\title{
CEESIt: A Computational Tool for the Interpretation of STR Mixtures
}

Harish Swaminathan ${ }^{1}$, Abhishek Garg ${ }^{2}$, Catherine M. Grgicak ${ }^{3}$, Muriel Medard ${ }^{4}$ and Desmond S. $\operatorname{Lun}^{1,2,5,6 . *}$

${ }^{1}$ Center for Computational and Integrative Biology, Rutgers University, Camden, NJ 08102, USA

${ }^{2}$ Department of Computer Science, Rutgers University, Camden, NJ 08102, USA

${ }^{3}$ Biomedical Forensic Sciences Program, Boston University School of Medicine, Boston, MA 02118, USA

${ }^{4}$ Research Laboratory for Electronics, Massachusetts Institute of Technology, Cambridge, MA 02139,

USA

${ }^{5}$ Department of Plant Biology and Pathology, Rutgers University, New Brunswick, NJ 08901, USA

${ }^{6}$ School of Information Technology and Mathematical Sciences, University of South Australia, Mawson

Lakes, SA 5095, Australia

* To whom correspondence should be addressed. Tel:[+1-856-225-6094]; Fax: [+1-856-225-6624];

Email:[dslun@rutgers.edu] Address: Department of Computer Science, Rutgers University, 227 Penn Street, Camden, NJ 08102, USA. 


\section{CEESIt: A Computational Tool for the Interpretation of STR} Mixtures

\section{ABSTRACT}

4 In forensic DNA interpretation, the Likelihood Ratio (LR) is often used to convey the strength of a 5 match. Expanding on binary and semi-continuous methods that do not use all of the quantitative data contained in an electropherogram, fully continuous methods to calculate the LR have been created. These fully continuous methods utilize all of the information captured in the

8 electropherogram, including the peak heights. Recently, methods that calculate the distribution of

9 the LR using semi-continuous methods have also been developed. The LR distribution has been

10 proposed as a way of studying the robustness of the $L R$, which varies depending on the

11 probabilistic model used for its calculation. For example, the LR distribution can be used to 12 calculate the $p$-value, which is the probability that a randomly chosen individual results in a LR 13 greater than the LR obtained from the person-of-interest (POI). Hence, the $p$-value is a statistic 14 that is different from, but related to, the LR; and it may be interpreted as the false positive rate 15 resulting from a binary hypothesis test between the prosecution and defense hypotheses. Here, 16 we present CEESIt, a method that combines the twin features of a fully continuous model to 17 calculate the LR and its distribution, conditioned on the defense hypothesis, along with an 18 associated $p$-value. CEESIt incorporates dropout, noise and stutter (reverse and forward) in its 19 calculation. As calibration data, CEESIt uses single source samples with known genotypes and 20 calculates a LR for a specified POI on a question sample, along with the LR distribution and a $p$ 21 value. The method was tested on 303 files representing 1-, 2- and 3- person samples injected 22 using three injection times containing between 0.016 and $1 \mathrm{ng}$ of template DNA. Our data allows 23 us to evaluate changes in the LR and $p$-value with respect to the complexity of the sample and to 24 facilitate discussions regarding complex DNA mixture interpretation. We observed that the 25 amount of template DNA from the contributor impacted the LR - small LRs resulted from 26 contributors with low template masses. Moreover, as expected, we observed a decrease of $p$ 27 values as the LR increased. A $p$-value of $10^{-9}$ or lower was achieved in all the cases where the 28 LR was greater than $10^{8}$. We tested the repeatability of CEESIt by running all samples in 29 duplicate and found the results to be repeatable.

\section{KEYWORDS}

31 Mixture interpretation, Likelihood ratio, $p$-value, DNA analysis, LR distribution

\section{INTRODUCTION}

Until recently, statements of inclusion or exclusion were exclusively used when reporting or presenting DNA comparisons to the trier-of-fact. If a suspect, or other known, is 'included' as a

35 potential contributor to the item of evidence, then the inclusion statement must be accompanied 
by the calculation of a statistic that conveys the strength of the match [1]. Alternatives to inclusion/exclusion statements have, of late, been adopted, where a verbal scale is used to

38 describe the number obtained [2].

39 Two protocols for calculating a match statistic are the Random Man Not Excluded (RMNE)

40 approach, based on the Combined Probability of Inclusion (CPI) statistic, and the Likelihood Ratio

41 (LR) approach. The RMNE method seeks to determine the fraction of the population that would

42 not be excluded as a contributor to the profile. During the calculation of the CPI statistic, some

43 information like the genotype of the suspect, the peak heights and the number of contributors to

44 the profile is not utilized [3].

45 Though RMNE is still employed in practice, this method of evaluation is being replaced with the

46 LR approach [4]. The Likelihood Ratio is defined as:

$$
L R=\frac{\operatorname{Pr}\left(E \mid H_{p}, n_{p}\right)}{\operatorname{Pr}\left(E \mid H_{d}, n_{d}\right)}
$$

where $E$ is the evidence in the form of the electropherogram (epg); $H_{p}$ and $H_{d}$ are the hypotheses specified by the prosecution and the defense, respectively; and $n_{p}$ and $n_{d}$ are the number of contributors specified by the prosecution and the defense, respectively. The Likelihood Ratio can be expressed either as the ratio of probabilities or as the ratio of probability densities, depending on whether the evidence is treated as a discrete or as a continuous random variable. The numerator is the probability of observing the evidence given the prosecution's hypothesis and the denominator is the probability of observing the evidence given the defense's hypothesis. The evidence shows support for the prosecution's hypotheses if $L R>1$; if $L R<1$ the defense's hypothesis is supported by the evidence. Unlike the RMNE method, the LR can use information like the number of contributors to the sample, the heights of the peaks observed and the genotype of the suspect [3].

The LR framework can be applied using a binary model that uses the set of alleles observed

60 in a DNA profile [5]. This method assigns a probability of 0 or 1 to genotypes based on the 61 presence or absence of alleles. Alternatives to the binary model have been proposed that allow

62 for drop-in and/or dropout of alleles [6,7]. These 'semi-continuous' methods use the peak heights

63 to establish probabilities of dropout and drop-in. Unlike binary methods, they can be used to

64 interpret profiles in which one or more of the suspect's alleles are not observed, or when there 65 are incidences of drop-in. Fully continuous methods that employ probabilistic genotyping by 66 modeling the peak heights have also been created, resulting in the ability to incorporate stutter 67 and noise, or drop-in, into the calculation of the statistic [8-12]. Fully continuous methods make 68 use of the entire data obtained in the epg, including the qualitative (alleles observed) and the 69 quantitative (peak heights) data. The TrueAllele system [8-9] uses an MCMC sampler to compute

70 a probability for every possible genotype combination based on how well it explains the observed 71 data. The peak heights are linearly modeled with respect to the mixture weights using a 
72 multivariate normal distribution. Degradation is modeled as an exponential decay with respect to 73 the allele product length and stutter as a linear function of the allele. Cowell et al [10] model the

74 peak heights using a Gamma distribution and employ a Bayesian network for analyzing mixtures

75 that incorporate dropout and a stutter model that is independent of DNA mass and the marker.

76 Puch-Solis et al [11] also use a gamma distribution for stutter and allele heights but differ from

77 [10] by using the total peak height at a locus as a proxy for the DNA mass, estimating parameters

78 conditional on peak heights and jointly modeling stutter and allelic peaks. Both these methods do

79 not take into account drop-in. Taylor et al [12] implement MCMC using the Metropolis-Hastings

80 algorithm to compute the genotype probabilities. Allele peak heights are modeled using an

81 exponential decay with respect to the molecular weight of the allele and stutter peak heights are

82 modeled as a linear function of the allele height. Drop-in is modeled as an exponential decay with

83 respect to the peak height.

84 In addition to the methods and models that evaluate LRs, computational methods that

85 compute the LR distribution have recently garnered attention [7,13-16]. The LR distribution can

86 be used to evaluate the robustness of the model by performing Tippett tests - "what is the

87 probability that a non-contributor will give rise to an LR greater than 1 (Type I error)?" [13].

88 Another statistic that can be obtained from the LR distribution is the $p$-value $[7,14-16]$. The $p$ -

89 value is a summary statistic that provides the probability that a randomly picked person from the

90 population would give rise to an LR at least as large as the one observed for the person of

91 interest. It can be interpreted as the false positive rate and may be useful when the analyst wants

92 to compute the probability of a random non-contributor giving rise to an LR greater than the one

93 observed for the suspect. While there is controversy surrounding the use of $p$-values [17], several

94 authors have shown that it is a useful statistic that assists in the interpretation of LRs and has

95 other applications like database searching [18] and kinship analysis [19].

96 In this work, we seek to combine the twin features of a fully continuous method to calculate

97 the LR and the calculation of the LR distribution and a $p$-value. To this end, we developed a

98 computational method called 'CEESIt' to calculate the LR for a person of interest, given an STR

99 profile. CEESIt (CEES: Computational Evaluation of Evidentiary Signal) is a fully continuous

100 method that works by modeling the peak heights observed in a calibration data set consisting of

101 single source samples with known genotypes. CEESlt accounts for dropout, noise and stutter

102 (both reverse and forward), artifacts observed regularly in low template samples [19]. Additionally,

103 CEESIt also computes a $p$-value for the LR by sampling a large number of random genotypes

104 from the population. The method was tested on 303, 1-, 2- and 3-person experimental sample

105 files with template masses ranging from 0.016 to $1 \mathrm{ng}$, and represents the largest empirical study

106 to evaluate the $p$-value and LR that we know of. We found that the amount of template DNA from

107 the contributor had an impact on the LR - small LRs were associated with low template masses.

108 Since we used $10^{9}$ samples to calculate the $p$-value, the lowest $p$-value that CEESIt reports is $10^{-}$ 
$109{ }^{9}$, and this was obtained in all the cases where the LR was greater than $10^{8}$. We also tested the

110 system's repeatability and found that the results were repeatable.

\section{MATERIALS AND METHODS}

\section{Calibration Set}

113 CEESIt employs a continuous method to calculate the LR and hence uses the peak heights in

114 the signal to calculate probabilities. Characterization of the peak heights is accomplished by using 115 single source calibration profiles with known genotypes obtained from samples amplified from a 116 wide range of input DNA masses. For a detailed description of how the calibration samples 117 (Calibration Set - Supplementary Table 1) were created, refer to [20]. Briefly, DNA was extracted 118 from 28 individuals. Absolute DNA quantification was performed using real-time PCR and the 119 Quantifiler $^{\circledR}$ Duo ${ }^{\text {TM }}$ Quantification kit according to the manufacturer's recommended protocol and 120 one external calibration curve [21, 22]. The extracted DNA was amplified using the 121 manufacturer's recommended protocol for AmpFlSTR ${ }^{\circledR}$ Identifiler ${ }^{\circledR}$ Plus Amplification Kit (Life 122 Technologies, Inc.) [23]. Separation of the STR fragments was accomplished with a 3130 Genetic 123 Analyzer using an injection voltage of $3 \mathrm{kV}$ and injection times of 5,10 and 20 seconds. Analysis 124 was performed using GeneMapper IDX v1.1.1 (Life Technologies, Inc.) and an RFU threshold of 125 1. A threshold of 1 RFU was used in order to capture all peak height information, i.e. the allelic 126 peaks, baseline noise and stutter peaks, in the signal. Known artifacts such as pull-up, spikes, $-\mathrm{A}$, 127 and artifacts due to dye dissociation were manually removed, as previously detailed in [20].

\section{Testing Set}

129 A total of 303 1-, 2- and 3-person sample files were used to test CEESIt (Testing Set 130 Supplementary Tables 2-4). These 1-person test samples were created using the same protocol 131 described for the single source samples in the calibration set. The mixtures were created by 132 mixing appropriate volumes of the single source DNA extracts to attain the various ratios 133 specified in Supplementary Table 5. Once mixed, these samples were re-quantified and then 134 amplified using the targets from Supplementary Tables 3 and 4 . In the case of mixtures, the 135 samples were created using various kinds of mixture ratios in such a way that each individual 136 contributed at least two cells' worth of DNA, which corresponds to approximately $0.013 \mathrm{ng}$ of DNA 137 [24]. The 1-person samples contained DNA from 32 different individuals, the 2-person samples 138 contained DNA from 6 different individuals (3 combinations) and the 3-person samples contained 139 DNA from 6 different individuals (2 combinations). When testing the samples injected for $5 \mathrm{sec}$, 140 the calibration file also consisted of data from $5 \mathrm{sec}$ injections. The same was true for the 10 and $14120 \mathrm{sec}$ injection files. None of the contributors to the calibration set were present in the testing set 142 and none of the contributors to the testing set were present in the calibration set.

\section{Modeling of variables}


144 For a detailed description of the model used for the various variables, see [20]. In short, the

145 allelic and noise peak heights were modeled using a Gaussian distribution. For stutter, since the

146 height of the stutter peak depends upon the height of the parent allele, the stutter ratio was

147 modeled using a Gaussian distribution. The allele frequencies used in this study were those of

148 the US Caucasian population published in [23], though any population database can be used.

149 The functions used to model the different variables with respect to the DNA mass were chosen by

150 fitting a curve to the calibration data in $\operatorname{MATLAB}^{\circledR}(\mathrm{R} 2015 \mathrm{~b}$, The Mathworks, Natick,

151 Massachusetts) and are shown in Table 1.

\section{CEESIt's Algorithm}

153 The Likelihood Ratio (LR) is defined as:

$$
L R=\frac{\operatorname{Pr}\left(E \mid H_{p}, n_{p}\right)}{\operatorname{Pr}\left(E \mid H_{d}, n_{d}\right)}
$$

154 The evidence is in the form of a discrete random variable that takes non-negative integer values

155 and CEESIt uses a continuous distribution, specifically the normal distribution, as the underlying

156 distribution from which a discrete distribution to model the peak heights is derived. Hence we

157 define the Likelihood Ratio to be the ratio of the two probabilities, rather than probability densities.

158 In practice, $n_{p}$ and $n_{d}$ can be chosen by the prosecution and the defense to maximize their

159 respective probabilities and there is no necessity for $n_{p}$ to be equal to $n_{d}$. However, we have

160 developed CEESIt to use the same number of contributors in both the numerator and

161 denominator to calculate the LR. It should be noted that the method could be extended to work on

162 different assumptions on the number of contributors. We note that for purposes of this work,

$163 n_{p}=n_{d}$ in all cases presented herein and we use the known, and thus the true $n$ to test the

164 capabilities of CEESIt, unless otherwise mentioned.

165 For this study, we use the following hypotheses for $H_{p}$ and $H_{d}$ :

$166 H_{p}$ : The evidence is a mixture of the genotype profile of a suspect and the profiles of $n-1$

167 other unknown, unrelated contributors, whom we term the 'interference' contributors.

$168 H_{d}$ : The evidence is from $n$ unknown individuals unrelated to the suspect.

169 It should be noted that the algorithm can be expanded to incorporate a victim or other known

170 profile in the prosecution and/or the defense hypothesis, but the current design of CEESIt allows

171 only for the genotype of a suspect to be included in the algorithm.

172 The LR for the suspect with genotype $s$ is:

$$
L R(s)=\frac{\operatorname{Pr}(E \mid R=s, N=n)}{\operatorname{Pr}(E \mid N=n)} .
$$

173 CEESIt's model incorporates a population substructure correction factor $\theta$. In this work, a value of 1740.01 was used for $\theta$.

175 LR numerator calculation: 
Our algorithm assumes a constant mixture ratio at all the loci. The mixture ratio specifies the proportion of the total template mass contributed by each contributor to the sample. The underlying mixture ratio of an evidence sample is unknown and needs to be described by a model in order to compute a continuous LR. A constant mixture ratio model assumes that the mixture ratio is the same at all the markers, whereas a variable mixture model accounts for the possibility

182 of the mixture ratio being different at the various markers. Both models are reasonable and are

183 used in existing continuous methods to compute the LR. Perlin et al [9] assign a uniform prior 184 probability for the template mixture weight and construct its probability distribution by drawing 185 individual locus weights using a multivariate normal distribution. Cowell et al [10] and Puch-Solis 186 et al [11] use a constant mixture ratio model and implement a discrete approximation over the interval $(0,1)$ by assigning a uniform prior. Taylor et al [12] use the variable model and assume the mixture weights to be independent across the loci. Since we adopt the constant mixture ratio approach, we integrate over all possible mixture ratios:

$$
\operatorname{Pr}(E \mid R=s, N=n)=\int_{\boldsymbol{\theta} \in \Delta^{n-1}} \operatorname{Pr}(E \mid \boldsymbol{\Theta}=\boldsymbol{\theta}, R=s, N=n) f_{\boldsymbol{\Theta}}(\boldsymbol{\theta}),
$$

where $\Theta$ is the vector with components $\Theta_{i}$, the mixture proportion of each contributor $i \in$ $\left\{1, \ldots, n_{\max }\right\} ; \Delta^{n-1}=\left\{\left(\Theta_{1}, \ldots, \Theta_{n}\right) \in \mathbb{R}^{n} \mid \sum_{i=1}^{n} \Theta_{i}=1, \Theta_{i}>0 \forall i\right\}$ is the unit $n-1$ simplex; and $f_{\Theta}$ is the probability density function of $\Theta$, which we assume to be uniform over $\Delta^{n-1}$. For $n=1, \Delta^{n-1}$ consists of the single element $\{1\}$. For mixtures, we implement the integration over $\Delta^{n-1}$ by dividing it into equal-sized subsets and representing each subset with its centroid, resulting in a discrete sum. This was done by performing k-means clustering in Python (Python Software Foundation, Beaverton, Oregon). k-means clustering is an algorithm used to partition observations into a set of clusters by repeated minimization of the distance from an observation to the centroid of its cluster [25]. For $n=2$, the space was divided into 9 equally sized clusters, while for $n=3,12$ clusters were used. See Supplementary Table 5 for a list of the mixture ratios used in CEESIt's algorithm. For the k-means algorithm, we used 10,000 randomly generated mixture ratios for $n=2$ and 100,000 randomly generated mixture ratios for $n=3$. The algorithm was stopped when there was no further possible minimization of the distance from a point to a centroid, i.e. when each point was assigned to the cluster with the closest centroid. Since the number of clusters ' $k$ ' is an input to the k-means clustering algorithm, we need to check if the choice made for the number of clusters is reasonable. To do this, we analyzed the effect of increasing the number of clusters on the probability that is conditional upon the mixture ratios. For this sensitivity test on the number of clusters, we used the forty-one 2-person samples and the thirty 3-person samples at the 10 s injection time that were part of our testing set. In these seventy-one samples, we computed $\operatorname{Pr}(E \mid R=s, N=n)$ for all the true contributors. We observed that increasing the number of clusters beyond our chosen values ( 9 for $n=2$ and 12 for $n=3$ ) 
211 had little effect on the probability (Supplementary Figures 1 and 2), indicating that the values

212 chosen are appropriate.

213 Let $\boldsymbol{L}$ be the set of all loci in the evidence sample, $E_{l}$ be the evidence at locus $l$ and $s_{l}$ be the 214 genotype of the suspect at locus $l$. The STR loci used for forensic DNA analysis are assumed to 215 be in linkage equilibrium and independent of each other [24]. Hence we obtain:

$$
\operatorname{Pr}(E \mid \boldsymbol{\Theta}=\boldsymbol{\theta}, R=s, N=n)=\prod_{l \in \boldsymbol{L}} \operatorname{Pr}\left(E_{l} \mid \boldsymbol{\Theta}=\boldsymbol{\theta}, R_{l}=s_{l}, N=n\right) .
$$

216 The prosecution's hypothesis states that the profile is made of the suspect's contribution plus the 217 contribution from $n-1$ other random, unrelated contributors. Therefore we get:

$$
\operatorname{Pr}\left(E_{l} \mid \boldsymbol{\Theta}=\boldsymbol{\theta}, R_{l}=s_{l}, N=n\right)=\sum_{\boldsymbol{u}^{n-1}} \operatorname{Pr}\left(E_{l} \mid \boldsymbol{U}^{n-1}=\boldsymbol{u}^{n-1}, \boldsymbol{\Theta}=\boldsymbol{\theta}, R_{l}=s_{l}, N=n\right) \operatorname{Pr}\left(\boldsymbol{U}^{n-1}=\boldsymbol{u}^{n-1}\right),
$$

218 where $\boldsymbol{u}^{i}=\left\{u_{1}, \ldots, u_{i}\right\}$ is a vector of the random genotypes of $i$ contributors. Since there are many 219 possibilities for the genotype of these interference contributors at each locus and summing over 220 each possibility would take a large amount of time, we approximate $\operatorname{Pr}\left(E_{l} \mid \boldsymbol{\Theta}=\boldsymbol{\theta}, R_{l}=s_{l}, N=n\right)$ 221 using importance sampling. Importance sampling is a Monte Carlo sampling algorithm in which, 222 instead of sampling directly from the target distribution, samples are generated from a different 223 distribution that is easier to sample from [26]. To take into account the fact that the samples have 224 come from the 'wrong' distribution, weights are introduced to adjust the 'importance' of each 225 sample. For the problem at hand, instead of sampling using the allele frequency distribution, we 226 generate samples of the interference genotypes using the peak height distribution observed at 227 the locus. The reason for sampling from the peak height distribution is that this method is faster 228 and requires fewer samples for convergence than the method that samples from the allele 229 frequency distribution.

230 Let $J$ be the number of interference samples used. Now we obtain:

$$
\operatorname{Pr}\left(E_{l} \mid \boldsymbol{\Theta}=\boldsymbol{\theta}, R_{l}=s_{l}, N=n\right) \approx \frac{\sum_{i=1}^{J} \operatorname{Pr}\left(E_{l} \mid \boldsymbol{U}_{i}^{n-1}=\boldsymbol{u}_{i}^{n-1}, \boldsymbol{\Theta}=\boldsymbol{\theta}, R_{l}=s_{l}\right) w_{i}}{J},
$$

231 where $w_{i}=P\left(\boldsymbol{u}_{i}^{n-1}\right) / Q\left(\boldsymbol{u}_{i}^{n-1}\right)$ is the weight of sample $i ; P\left(\boldsymbol{u}_{i}^{n-1}\right)$ is the probability of the 232 interference genotypes under the allele frequency distribution; and $Q\left(\boldsymbol{u}_{i}^{n-1}\right)$ is the probability of 233 the interference genotypes under the peak height distribution.

234 Importance sampling is done for autosomal loci, which are based on STRs. The locus AMEL, the 235 sex-determining locus, is not based on STRs and has only 2 possible genotypes: $\{X, X\}$ for 236 females and $\{X, Y\}$ for males. At this locus, we assume that both genotypes are equally likely to 237 occur and generate genotype samples for the $n$ contributors and calculate the probability of 238 observing the evidence as:

$$
\operatorname{Pr}\left(E_{l} \mid \boldsymbol{\Theta}=\boldsymbol{\theta}, R_{l}=s_{l}, N=n\right) \approx \frac{\sum_{i=1}^{J} \operatorname{Pr}\left(E_{l} \mid \boldsymbol{U}_{i}^{n-1}=\boldsymbol{u}_{i}^{n-1}, \boldsymbol{\Theta}=\boldsymbol{\theta}, R_{l}=s_{l}\right)}{J} .
$$


239 Calculation of $\operatorname{Pr}\left(E_{l} \mid \boldsymbol{U}_{i}^{n-1}=\boldsymbol{u}_{i}^{n-1}, \boldsymbol{\Theta}=\boldsymbol{\theta}, R_{l}=s_{l}\right)$ is explained in the Appendix as part of the

240 Supplementary Material.

\section{LR distribution and $\mathrm{p}$-value calculation:}

The $p$-value for the suspect is defined as the probability that a randomly picked person from the population would give rise to an LR at least as large as the one observed for the suspect.

$$
p \text {-value }(s)=\operatorname{Pr}(L R(R) \geq L R(s)) \text {, }
$$

246 where $R$ is a random variable denoting the genotype of an individual. Since the denominator of 247 the LR is the same for all genotypes, it is sufficient if we compare the numerator of the LR for $R$ 248 and $s$.

$$
p \text {-value }(s)=\operatorname{Pr}(\operatorname{Pr}(E \mid R, N=n) \geq \operatorname{Pr}(E \mid R=s, N=n)) .
$$

249 During testing of CEESIt, we observed that because of floating-point precision, $\operatorname{Pr}(E \mid R, N=n)$

250 evaluated to 0 for many of the random genotypes $R$ that fit the data poorly. As a result, we were 251 able to eliminate those genotypes from the $p$-value calculation as a preliminary step. Formally, let

$252 \boldsymbol{R}$ be the set of all genotypes. We define $\boldsymbol{R}_{\mathbf{1}}=\left\{r \in \boldsymbol{R} \mid \operatorname{Pr}\left(E_{l} \mid R_{l}=r_{l}\right) \nsim 0\right.$ for all loci $\left.l\right\}$ and

$253 \boldsymbol{R}_{\mathbf{2}}=\left\{r \in \boldsymbol{R} \mid \exists\right.$ locus $l$ s.t. $\left.\operatorname{Pr}\left(E_{l} \mid R_{l}=r_{l}\right) \simeq 0\right\}$, where $\simeq 0$ means "evaluates to 0 using double-

254 precision 64-bit floating-point arithmetic". Thus, we have $\mathbf{R}=\boldsymbol{R}_{\mathbf{1}} \cup \boldsymbol{R}_{\mathbf{2}}$ and $\boldsymbol{R}_{\mathbf{1}} \cap \boldsymbol{R}_{\mathbf{2}}=\emptyset$. We see

255 that for all $r \in \boldsymbol{R}_{2}, \operatorname{Pr}(E \mid R=r) \simeq 0$. We omit the notation on $N$ for the sake of brevity. We have:

$$
\begin{aligned}
p \text {-value }(s)=\left\{\begin{array}{l}
\operatorname{Pr}\left(R \in \boldsymbol{R}_{\mathbf{1}}\right) \\
\sum_{r \in \boldsymbol{R}_{\mathbf{1}}} \operatorname{Pr}(\operatorname{Pr}(E \mid R=r) \geq \operatorname{Pr}(E \mid R=s)) \operatorname{Pr}\left(R=r \mid R \in \boldsymbol{R}_{\mathbf{1}}\right)
\end{array}\right. \\
\left.\quad+\operatorname{Pr}\left(R \in \boldsymbol{R}_{\mathbf{2}}\right) \sum_{r \in \boldsymbol{R}_{\mathbf{2}}} \operatorname{Pr}(\operatorname{Pr}(E \mid R=r) \geq \operatorname{Pr}(E \mid R=s)) \operatorname{Pr}\left(R=r \mid R \in \boldsymbol{R}_{\mathbf{2}}\right) .\right\}
\end{aligned}
$$

256 We see that the second term is 0 , provided $\operatorname{Pr}(E \mid R=s)$ is greater than 0 . Hence we get:

$$
p \text {-value }(s)=\operatorname{Pr}\left(R \in \boldsymbol{R}_{\mathbf{1}}\right) \sum_{r \in \boldsymbol{R}_{\mathbf{1}}} \mathbf{1}(\operatorname{Pr}(E \mid R=r) \geq \operatorname{Pr}(E \mid R=s)) \operatorname{Pr}\left(R=r \mid R \in \boldsymbol{R}_{\mathbf{1}}\right),
$$

257 where

$$
\mathbf{1}(\operatorname{Pr}(E \mid R=r) \geq \operatorname{Pr}(E \mid R=s))=\left\{\begin{array}{c}
1, \text { if } \operatorname{Pr}(E \mid R=r) \geq \operatorname{Pr}(E \mid R=s) \\
0, \text { otherwise }
\end{array}\right.
$$

259 We have:

$$
\operatorname{Pr}\left(R \in \boldsymbol{R}_{\mathbf{1}}\right)=\prod_{l \in \boldsymbol{L}} \sum_{\boldsymbol{r}_{l} \in\left\{\boldsymbol{r} \mid \operatorname{Pr}\left(E_{l} \mid R_{l}=r\right) \neq 0\right\}} \operatorname{Pr}\left(R_{l}=r_{l}\right) .
$$

260 We compute the $p$-value using Monte Carlo simulation. We generate $M$ random genotypes

$261 r^{1}, \ldots, r^{M}$ according to the distribution $\operatorname{Pr}\left(R \mid R \in \boldsymbol{R}_{\mathbf{1}}\right)$ and calculate the $p$-value as:

$$
p \text {-value }(\mathrm{s})=\operatorname{Pr}\left(R \in \boldsymbol{R}_{\mathbf{1}}\right) \frac{\sum_{i=1}^{M} \mathbf{1}\left(\left(\operatorname{Pr}\left(E \mid R=r^{i}\right) \geq \operatorname{Pr}(E \mid R=s)\right)\right.}{M} .
$$

262 Increasing the value of $M$ increases the accuracy of the $p$-value computed, but this also increases 
263 the run time and a hence a tradeoff has to be achieved between the two. In this study, we have 264 used 1 billion or $10^{9}$ random genotypes to compute the $p$-value.

265 In some cases, $\sum_{i=1}^{M} \mathbf{1}\left(\left(\operatorname{Pr}\left(E \mid R=r^{i}\right) \geq \operatorname{Pr}(E \mid R=s)\right)\right.$ evaluated to 0 when no simulated

266 genotype had an LR at least as large as the POl's. This means that the $p$-value for the POI is less

267 than $\operatorname{Pr}\left(R \in \boldsymbol{R}_{\mathbf{1}}\right) / M$ and more samples are required for estimation. As a result, we could only

268 arrive at an upper bound on the $p$-value for such samples since we calculate the $p$-value by

269 sampling and do not perform an exact computation. Since $\operatorname{Pr}\left(R \in \boldsymbol{R}_{\mathbf{1}}\right)$ in general varies with the

270 sample, the upper bound also in general varies from one sample to another. Hence, to maintain

271 consistency in our representation of the CEESIt results, whenever the $p$-value evaluated to less

272 than $1 / M$ for a contributor, we report $1 / M$ or $10^{-9}$ as an upper bound on the $p$-value.

273 In order to facilitate the computation of the $p$-value, as an initial step $\operatorname{Pr}\left(E_{l} \mid \boldsymbol{\Theta}=\boldsymbol{\theta}, R_{l}=g_{l}\right)$ is

274 computed for all possible genotypes $g_{l}$ at all loci $l$ for all values of $\boldsymbol{\theta}$. Once this is done, for the $p$ -

275 value computation, $10^{9}$ genotypes $r^{i}$ are generated based on the allele frequencies. Since we

276 know $\operatorname{Pr}\left(E_{l} \mid \boldsymbol{\Theta}=\boldsymbol{\theta}, R_{l}=r_{l}^{i}\right)$ at all loci $l$, we can compute $\operatorname{Pr}\left(E \mid R=r^{i}\right)$ as:

$$
\operatorname{Pr}\left(E \mid R=r^{i}\right)=\int_{\boldsymbol{\theta} \in \Delta^{n-1}} \prod_{l} \operatorname{Pr}\left(E_{l} \mid \boldsymbol{\Theta}=\boldsymbol{\theta}, R_{l}=r_{l}^{i}\right) f_{\boldsymbol{\Theta}}(\boldsymbol{\theta}) .
$$

\section{LR denominator calculation:}

278 Let $\bar{R}$ be the genotype of an unknown contributor in the defense's hypothesis. The

279 denominator of the LR can be written as:

$$
\operatorname{Pr}(E \mid N=n)=\sum_{\bar{r}} \operatorname{Pr}(E \mid \bar{R}=\bar{r}, N=n) \operatorname{Pr}(\bar{R}=\bar{r})
$$

280 Since the number of possible values that $\bar{r}$ can take is large and summing over all of them is

281 computationally intensive, we utilize the calculations done for the random genotypes $r^{i}$ that are

282 sampled for the $p$-value estimation to compute the denominator of the LR as:

$$
\operatorname{Pr}(E \mid N=n)=\operatorname{Pr}\left(R \in \boldsymbol{R}_{\mathbf{1}}\right) \frac{\sum_{i=1}^{M} \operatorname{Pr}\left(E \mid R=r^{i}\right)}{M} .
$$

\section{RESULTS}

284 Contributors with $L R$ less than 1 have low template DNA masses

285 Figure 1 shows LR vs contributor template mass for all the contributors for 101, 1-, 2- and 3-

286 person samples at the 10 s injection in the testing set. The LRs for the 5 s and the 20 s samples

287 were similar to the 10 s samples and are shown in Supplementary Figures 3 and 4 . All the log

288 values shown in this study are to base 10. For each sample, the LR was computed for all the

289 contributors. Hence, a single source sample would have $1 \mathrm{LR}$; a 2-person sample would have 2

290 LRs, etc. We observed that the amount of template DNA from the contributor impacted the LR 
291 from CEESIt, i.e. high LRs corresponded to high template DNA amounts. This effect was

292 previously reported by Taylor et al [12]. While most $\log (\mathrm{LR})$ values in Figure 1 are greater than 0

293 (the highest was 100, with a mean of 15), CEESIt computed the log(LR) as less than 0 (or LR <

294 1) for a contributor in 7 cases (two 1-person samples and five 3-person samples). These samples

295 had a low template DNA from the contributor and a high level of dropout and stutter.

296 For example, a 3-person sample with $0.59 \mathrm{ng}$ of template DNA and a mixture ratio of 1:9:9

297 resulted in a $\log (\mathrm{LR})$ of -7 for the minor contributor ( $0.03 \mathrm{ng}$ template mass per qPCR

298 quantification). Figure 2 shows the profile of the sample and Table 2 shows the genotype of the 3

299 contributors. Supplementary Table 6 contains the heights of the peaks in Figure 2. One of

300 Contributor 1's alleles completely dropped out at 3 loci: allele 8 at D13S317, allele 20 at D2S1338

301 and allele 18 at vWA. Moreover, at locus D18S51 the forward stutter (height of 10 RFU) from

302 allele 17 has a larger height than the parent peak (height of 9 RFU). These factors caused

303 CEESIt to compute a low probability for the minor contributor. On the other hand, the alleles of

304 major contributors 2 and 3 ( $0.28 \mathrm{ng}$ template mass each) are well represented in the epg

305 resulting in a LR of $10^{12}$ and $10^{11}$, respectively. The distribution of the LRs for the 1 billion random

306 genotypes simulated for this sample is seen in Figure 3. A vast majority of the 1 billion samples

307 had a $\log (\mathrm{LR})$ less than 0 , with a peak around -30 . The LRs for the 3 contributors were near the

308 right end of the distribution; the resulting $p$-values were $7 \times 10^{-4}$ for the minor contributor and 10

$309{ }^{9}$ for the two major contributors.

310 When dropout levels were not high and the alleles were well represented in the epg, even minor

311 contributors had high LRs. Figure 4 shows the profile of a 2-person mixture, with $0.25 \mathrm{ng}$ of

312 template DNA and a 1:2 mixture ratio. Supplementary Table 7 contains the heights of the peaks

313 in Figure 4. The genotypes of the 2 contributors can be seen in Table 3. All the alleles of the

314 contributors can be seen in the profile and the LR was $10^{19}$ for both contributors. Figure 5 shows

315 the LR distribution for the 1 billion random genotypes simulated for this sample. Most of the

316 samples had a $\log (\mathrm{LR})$ less than 0 . The LRs for the contributors to the sample were in the right

317 tail of the distribution; the $p$-value was $10^{-9}$ for the major and the minor contributor.

318 The Random Match Probability (RMP) is an inclusion statistic that stems from a binary approach,

319 i.e. alleles are either present or absent [19]. The statistic is calculated using only the frequencies

320 of the alleles observed. We computed the RMP for the single source samples using an analytical

321 threshold of 50 RFU and the stutter filter and the US Caucasian allele frequencies published in

322 [23]. In our testing set, 34 out of the 91 single source files resulted in a 'match' and hence a RMP

323 was computed for the 34 samples. For all those 34 samples, the LR from CEESIt was similar to 1

324 / RMP [1] - the absolute value of the difference between $\log (\mathrm{LR})$ and $\log (1 / \mathrm{RMP})$ was less than 1

325 in 26 out of the 33 samples, with the lowest value being 0.109 . The largest difference observed

326 between $\log (\mathrm{LR})$ and $\log (1 / \mathrm{RMP})$ was 16 , with $\log (\mathrm{LR})$ being smaller. 
328 There were two contributors to the 1-person samples who were also minor contributors in 2-

329 person and 3-person samples in the testing set. We observed that for a given template DNA

330 mass, the LR was highest for the 1-person sample and decreased in the presence of an

331 interference contributor in a 2-person sample with a 1:1 mixture ratio. The LR further decreased

332 with 2 interference contributors in a 3-person sample with a 1:2:1 mixture ratio (Figure 6). We did

333 observe that at all three injection times, for some 2-person samples the $\log (\mathrm{LR})$ for the minor and

334 major contributors was large, and even larger than the $\log (\mathrm{LR})$ for some 1-person samples. We

335 found that the log(LR)'s were large in these instances because the two contributors gave the

336 best, or close to the best, fit to the signal and the denominator of the LR had a very low

337 probability.

338 Large LRs correspond to small p-values

339 Figure 7 (a) shows how the $p$-values from CEESIt vary with LR for the samples at the 10s 340 injection. In general, as the LR increased in value, the $p$-value decreased, indicating a greater 341 confidence in including the individual as a contributor. All the 1-person samples had the lowest 342 possible $p$-value of $10^{-9}$. Even the 1 -person samples that had a LR $<1$ had a $p$-value of $10^{-9}-$ 343 though their probability was low because of high levels of dropout and stutter, it was still high 344 relative to the population. For the 2-person and 3-person samples, in all cases where the $\log (\mathrm{LR})$ 345 was greater than 8 , the $\log (p$-value) from CEESIt was -9 , indicating that CEESIt was able to 346 identify the individual as a contributor with great certainty. Figure 7 (b) shows the same plot 347 zoomed in on the $\log (\mathrm{LR})$ values between 0 and 9. All the $\log (p$-value) points represent 2- and 3348 person samples and they lie below the line representing $\log (\mathrm{LR})$. This relationship between the $349 p$-value and the LR is expected because the $p$-value $\leq 1 /$ LR according to Markov's inequality 350 [27]. The 5 s and the 20 s samples showed a trend similar to the 10 s samples (Supplementary 351 Figures 5 and 6 ).

352 LRs for non-contributors are low

353 As described in Materials and Methods, for each of the 303 sample files in our testing set, $\boldsymbol{R}_{\mathbf{1}}$ 354 contains all the genotypes with a non-0 probability. CEESIt produced the LR distribution for a 355 sample by sampling the genotypes of $10^{9}$ random contributors from the set $\boldsymbol{R}_{\mathbf{1}}$. This resulted in a 356 total of $303 \times 10^{9} \approx 3 \times 10^{11}$ random genotypes for which we obtained a LR from CEESIt. The LR 357 distribution for every sample was similar to the ones shown in Figures 3 and 5 , with a majority of 358 the LRs falling below 1 . For each sample, we calculated the fraction of the $10^{9}$ genotypes that 359 resulted in a $L R>1$ and multiplied it by $\operatorname{Pr}\left(R \in \boldsymbol{R}_{\mathbf{1}}\right)$ to calculate the Tippet statistic $\operatorname{Pr}\left(L R>1 \mid H_{d}\right)$.

360 We observed that the average of the Tippett statistic increased as the number of contributors to 361 the sample increased, indicating that the probability of a random non-contributor having a $L R>1$ 362 is low for small $n$ and increases as $n$ increases. The average $\operatorname{Pr}\left(L R>1 \mid H_{d}\right)$ for the 1-person 363 samples was $2.34 \times 10^{-12}$ and it increased to 0.0004 and 0.0008 for the 2 - and 3-person samples. 
364 The smallest LR observed for the random contributors was 0 , while the maximum was $10^{31}$ (for a

365 1-person sample in which the true contributor to the sample had a LR of $10^{31}$ ).

366 The results from CEESIt are repeatable

367 One of the consequences of using a sampling algorithm (like importance sampling or Monte Carlo

368 simulation) is that the results of two analyses on the same sample are generally not identical.

369 While increasing the number of samples reduces the run-to-run variation, doing so also increases

370 the running time. Hence a trade-off between the two has to be achieved. We ran CEESIt on all

371 the samples in the testing set twice to check if the results were repeatable, i.e. if the LRs from the

372 two runs were similar to each other. Figure 8 shows the results on the 101, 1-, 2- and 3-person

373 samples at the 10 s injection. There is very little variation from run to run; the points lie close to the

$374 x=y$ diagonal line with very few outliers (Correlation coefficient $=0.9905$, slope $=1.04$, intercept $=$

$375-0.1$ ). Additionally we tested repeatability of CEESIt on one 2-person sample and one 3-person

376 sample after 5 runs and found that the LRs and $p$-values obtained were repeatable

377 (Supplementary Figures 7 and 8 ). Tests for repeatability on the 5 s and the 20 s samples also

378 produced similar results (Supplementary Figures 9 and 10).

379

380

Sensitivity to the number of contributors

381

382

Since the true number of contributors to an evidence sample is never known with certainty, we examined the performance of CEESIt when conditioned upon different assumptions on the number of contributors. For this sensitivity study on the number of contributors, we focus on the samples at the 10 s injection time that were incorrectly called by the maximum allele count (MAC) method. MAC is a binary method to estimate the number of contributors to a sample [28]. It works by counting the number of alleles observed at every locus, taking the maximum value over all the loci and dividing it by 2 to get the minimum number of contributors needed to explain the profile. MAC works by the application of an analytical threshold and a stutter threshold. Peaks below the analytical and the stutter thresholds are labeled as noise and stutter, respectively, and peaks above the thresholds are considered as signal and used in the calculation. In this study, we used an analytical threshold of 50 RFU, which is commonly used in forensic laboratories, and the stutter threshold for each locus as specified in [23].

397 This effect was more pronounced for the 2-person sample than for the 3-person samples. Except 398 for one contributor in one of the 3-person samples, all the other LRs using an incorrect number of 399 contributors were less than 1 (Figure 9). 
400 There were 3 overestimates among the 1-person samples and 2 overestimates among the 2-

401 person samples, all of which were called as one more than actual number by the MAC method.

402 Although MAC typically gives the minimum number of contributors needed to explain the profile,

403 these overestimates occurred due to stutter peaks that exceeded the stutter threshold and hence

404 were wrongly labeled as signal peaks. Using an overestimate on the number of contributors,

405 unlike underestimates, did not result in a large decrease in the LR. For the 1-person samples,

406 using an overestimating the number of contributors resulted in a similar LR and the LR even

407 slightly increased for one of the samples. For the 2-person samples which were analyzed as 3-

408 person mixtures, the LR did decrease, though it was greater than 1 (Figure 10).

409 These results suggest that the output from CEESIt is indeed sensitive to the number of

410 contributors specified and a good estimate on the number is crucial for interpretation of the

411 sample.

412

413 Runtime

414 The running time of CEESIt increases as the number of contributors increases. For single 415 source samples, the average running time was 7 minutes, and it increased to 50 and 140 minutes

416 for 2- and 3-person samples, respectively. CEESIt has been implemented to employ multi-

417 threaded processing to take advantage of multiple cores when available. The calculations were

418 done using 8 cores on an Intel E3 3.4GHz processor.

\section{DISCUSSION}

420 We have developed a computational algorithm - CEESIt - to compute the LR for a POI and the 421 distribution of the LR given an evidence profile. CEESIt also provides a summary statistic of the 422 distribution in the form of the $p$-value of the LR. There is no 'correct' value for the LR to compare

423 the results with - the LR computed is dependent upon the model used. Hence, we need to

424 perform tests to check if the results from the model are reasonable, i.e. if CEESIt computes a

425 'large' LR in the case of a true contributor to the mixture and a 'small' LR for a non-contributor.

426 CEESIt was tested on 303 sample files containing between 1 and 3 contributors with total

427 template mass ranging from $0.016 \mathrm{ng}$ to $1 \mathrm{ng}$, injected using 3 injection times. Across all samples

428 tested, the mean of the LRs was $10^{14}$; the lowest and highest LRs were $10^{-74}$ and $10^{100}$

429 respectively for minor (or single) contributors ranging in mass from $\sim 0.013$ to $0.33 \mathrm{ng}$. We

430 observed that the large LRs were obtained in instances where the LR denominator was small and

431 the true contributor to the sample gave the best (or close to the best) fit for the evidence. For

432 example, a LR of $10^{100}$ was obtained for a 1-person sample at the 10 s injection with $0.0156 \mathrm{ng}$ of

433 template DNA. The numerator of the LR evaluated to $10^{-135}$. The contributor's genotype gave the

434 best, or close to the best, explanation for the evidence and the denominator of the LR was

435 extremely small $\left(10^{-235}\right)$, resulting in the large LR. Such large LRs are typically not obtained from 
436 binary interpretation methods such as [5], which take into account only the alleles observed and 437 ignore other information like the heights of the peaks in the signal. In instances where CEESIt

438 produced a small LR, the template DNA mass from the contributor was low, resulting in extreme 439 dropout and stutter.

440 We observed that the presence of additional contributors impacted the LR for a POI. Specifically, 441 we observed that the addition of interference contributors decreased the LR for a POI that 442 contributed the same template mass to a 1-, 2- and 3-person sample, where, the LR was highest 443 for the 1-person sample, followed by the 2-person sample and then the 3-person sample.

444 For comparison, we calculated a binary inclusion statistic for the single source samples in the 445 form of the RMP. We observed that for samples for which we could calculate a RMP, the LR from 446 CEESIt was close to 1 / RMP, as expected. The smallest difference observed between log(LR) 447 and $\log (1 / \mathrm{RMP})$ was 0.109 , while the largest difference was 16 , with $\log (\mathrm{LR})$ being smaller.

448 In order to compare CEESIt with other published results we focus on 2-person mixture cases. We 449 note that in order to test CEESIt, the template masses used for the 2-person samples set in this 450 studied varied from 0.2 to $1 \mathrm{ng}$ and the mixture ratios varied from $1: 1$ to $1: 49$, where the lowest 451 DNA mass of the minor contributed $\sim 0.013 \mathrm{ng}$ of DNA. For all of the 122 2-person mixture files 452 tested in this work, the LR ranged from $10^{-63}$ to $10^{79}$. Taylor et al [12] performed a similar study 453 using an alternative continuous interpretation scheme and observed LRs in the range $10^{8}$ to $10^{23}$ 454 on 127 artificially constructed 2-person mixtures where the total template mass varied from 0.1 to $455 \quad 0.5 \mathrm{ng}$ and the mixture ratios from $1: 1$ to $1: 5$, resulting in a minor contributor mass of at least $4560.017 \mathrm{ng}$. TrueAllele [9] was tested on 101 casework profiles containing between 2 and 4 457 contributors. The mixture weight for the matched genotype varied from 0.05 to 0.95 . The LRs 458 ranged from 10 to $10^{23}$, with a mean of $10^{11}$.

459 In addition to calculating the LR, CEESIt also makes available to the user a distribution on the LR. 460 The LR distribution can be useful while performing error rate calculations such as

$461 \operatorname{Pr}\left(L R>1 \mid H_{d}\right)$ [13]. CEESIt also provides a summary statistic of the LR distribution in the form of 462 the $p$-value. The $p$-value, which can be thought of as the false positive rate, is the probability that 463 a randomly picked person results in a LR greater than the LR observed for the POI. In this study, 464 CEESIt calculated the $p$-value by generating $10^{9}$ random genotypes according to the allele 465 frequency distribution and computing the fraction of those genotypes that have a LR greater than 466 the POI's LR. All the 1-person samples in the testing set had a $p$-value of $10^{-9}$ or lower, indicating 467 that CEESIt was able to identify the individual as a contributor with great certainty. With regard to 468 the 2- and 3-person samples, the $p$-value was the lowest possible value of $10^{-9}$ when the LR was 469 greater than $10^{8}$.

470 One desirable quality of a mixture interpretation method is specificity: How often would a method 471 misidentify a non-contributor? In our testing, CEESIt produced a LR distribution for a test sample 472 by sampling $10^{9}$ genotypes, giving $\sim 3 \times 10^{11}$ random genotypes with a LR from CEESlt. For each 
473 experimental sample, we calculated the Tippett test statistic $\operatorname{Pr}\left(L R>1 \mid H_{d}\right)$ [13]. We observed

474 that the probability of a random non-contributor resulting in a $L R>1$ is low for small $n$ and

475 increases as $n$ increases. The average $\operatorname{Pr}\left(L R>1 \mid H_{d}\right)$ for the 1 -person samples was $2.34 \times 10^{-12}$

476 and it increased to 0.0004 and 0.0008 for the 2- and 3-person samples, respectively. The

477 minimum LR observed for the random contributors (sampled over the set $\boldsymbol{R}_{\mathbf{1}}$, see Materials and

478 Methods) was 0 , while the maximum was $10^{31}$. Perlin et al [9] tested TrueAllele by computing the

479 LR for 10,000 random contributors on each of the 101 samples in their testing set and found 133

480 instances in which the LR was greater than 1. The highest LR was $10^{4}$ and lowest was $10^{-30}$.

481 Similarly, Taylor et al observed a maximum LR of $10^{4}$ and 1168 LRs greater than 1 after testing

48257,609 non-contributors on a 3-person mixture [12].

483 Another desirable aspect of a sampling algorithm is reproducibility from one run to the next on the

484 same sample, i.e. obtaining similar results. CEESIt was run twice on all the samples in the testing

485 set and the results were repeatable (The correlation coefficient was greater than 0.9 for the

486 samples at all 3 injection times). Additionally, testing on one 2-person and one 3-person sample

487 confirmed the repeatability of CEESIt after 5 runs. TrueAllele variability from run to run was tested

488 on duplicate runs and on average the LR varied by a factor of $2\left(10^{0.305}\right)$ standard deviations [9].

489 Taylor et al [12] showed their model to give reproducible results over 10 runs on a single 2-

490 person mixture, with a standard deviation of $\sim 3 \%$ of the mean.

\section{CONCLUSION}

492 Interpretation of low template samples is a challenging problem. Lately there has been a push 493 towards the LR as the method of choice for calculating a match statistic. Fully continuous

494 methods to calculate the LR have the benefit of making use of all the information contained in the 495 signal.

496 One question that forensic analysts are interested in is "How many times would a non-contributor

497 come up with a statistic that is as large or larger?" or in other words, "What is the false positive

498 rate?" In addition to the LR, calculation of the LR distribution and a resulting summary statistic -

499 the $p$-value - has been reported, with the $p$-value serving as a false positive rate. To date, the

500 only systems available to compute both the LR and $p$-value used semi-continuous models.

501 In this work, we have brought together a continuous method to compute the LR based on

502 modeling of the peak heights in known data and to calculate a $p$-value from the LR distribution by

503 simulation of genotypes based on allele frequencies in order to demonstrate, using a large set of

504 data, that systems which determine both a $p$-value and an LR are computationally viable. We do

505 not propose to prescribe the use of one statistic over the other for purposes of reporting or

506 testimony. Rather, we describe a study that examines the impact of the complexity of the sample

507 on both statistics using a fully continuous interpretation scheme. The method was tested on 303

508 1-, 2- and 3-person sample files containing between 0.016 and $1 \mathrm{ng}$ of DNA, injected for 5,10 
509 and $20 \mathrm{sec}$. We found that the amount of template DNA from the contributor had an impact on the

510 LR - small LRs arose from contributors with low template masses, indicating that high levels of

511 dropout and stutter could decrease the probability of the evidence under the prosecution's

512 hypothesis even for true contributors. Since we used $10^{9}$ samples to calculate the $p$-value, the

513 lowest $p$-value that we report is $10^{-9}$, and this was obtained in all the cases where the LR was

514 greater than $10^{8}$. The results from CEESIt were found to be repeatable after duplicate runs on all

515 the samples in the testing set. Even though we used low-medium template masses in our study,

516 CEESIt can be tested on unknown samples with any template mass, provided the mass falls

517 within the range of the masses of the calibration samples.

518 While we have compared the results from CEESIt with the published results of other continuous

519 interpretation software, comparison of the results from CEESIt with other continuous

520 interpretation methods on the same set of data is important and would facilitate standardization of

521 validation approaches. Future work involves developing CEESlt to handle different numbers of

522 contributors in the prosecution and the defense hypotheses and accounting for known (and

523 possibly related) contributors. In a follow up paper, we plan on testing the sensitivity of the LR to

524 the changes in the model. Information on downloading and using CEESIt can be found at

525 www.bu.edu/dnamixtures.

\section{FUNDING}

527 This project was partially supported by NIJ2011-DN-BX-K558, NIJ2012-DN-BX-K050 and

528 NIJ2014-DN-BX-0001 awarded by the National Institute of Justice, Office of Justice Programs,

529 U.S. Department of Justice and NSF1126052 awarded by the National Science Foundation. The

530 opinions, findings, and conclusions or recommendations expressed in this

531 publication/program/exhibition are those of the author(s) and do not reflect those of the

532 Department of Justice or the National Science Foundation.

\section{ACKNOWLEDGEMENT}

534 The authors would like to thank Lauren Alfonse and Genevieve Wellner of Boston University for

535 their help with the preparation of the samples used in this study.

\section{REFERENCES}

537 1. SWGDAM. (2010). SWGDAM interpretation guidelines for autosomal STR typing by forensic

538 DNA testing laboratories. Available at: http://www.swgdam.org.

539 2. ENFSI. (2015). ENFSI guideline for evaluative reporting in forensic science: Strengthening the

540 Evaluation of Forensic Results across Europe. Available at: http://www.enfsi.eu.

541 3. J. Buckleton, J. Curran, A discussion of the merits of random man not excluded and likelihood 
542 ratios, Forensic Sci Int Genet. 2 (2008) 343-348. DOI: 10.1016/j.fsigen.2008.05.005.

543 4. P. Gill, L. Gusmão, H. Haned, W.R. Mayr, N. Morling, W. Parson, L. Prieto, M.Prinz, H.

544 Schneider, P.M. Schneider, B.S. Weir, DNA commission of the International Society of Forensic

545 Genetics: Recommendations on the evaluation of STR typing results that may include drop-out

546 and/or drop-in using probabilistic methods, Forensic Sci Int Genet. 6 (2012) 679-688. DOI:

$547 \quad$ 10.1016/j.fsigen.2012.06.002.

548 5. H. Kelly, J. Bright, J. Curran, J. Buckleton, The interpretation of low level DNA mixtures,

549 Forensic Sci Int Genet. 6 (2012) 191-197. DOI: 10.1016/j.fsigen.2011.04.013.

550 6. D. Balding, J. Buckleton, Interpreting low template DNA profiles, Forensic Sci Int Genet. 4

551 (2009) 1-10. DOI: 10.1016/j.fsigen.2009.03.003.

552 7. P. Gill, H. Haned, A new methodological framework to interpret complex DNA profiles using

553 likelihood ratios, Forensic Sci Int Genet. 7 (2013) 251-263. DOI: 10.1016/j.fsigen.2012.11.002.

554 8. M.W. Perlin, M.W. Legler, C.E. Spencer, J.L. Smith, W.P. Allan, J.L. Belrose, B.W. Duceman, 555 Validating TrueAllele DNA Mixture Interpretation. J Forensic Sci. 56:6 (2011) 1430-1447. DOI:

556 10.1111/j.1556-4029.2011.01859.x.

557 9. M. W. Perlin, K. Dormer, J. Hornyak, L. Schiermer-Wood, S. Greenspoon, TrueAllele

558 Casework on Virginia DNA Mixture EvidenceL Computer and Manual Interpretation in 72

559 Reported Criminal Cases. PLoS ONE 9 (2014). DOI: 10.1371/journal.pone.0092837.

560 10. R.G. Cowell, S.L. Lauritzen, J. Mortera, Probabilistic expert systems for handling artifacts in 561 complex DNA mixtures, Forensic Sci Int Genet. 5 (2011) 202-209. DOI:

$562 \quad$ 10.1016/j.fsigen.2010.03.008.

563 11. R. Puch-Solis, L. Rodgers, A. Mazumder, S. Pope, I. Evett, J. Curran, D. Balding, Evaluating 564 forensic DNA profiles using peak heights, allowing for multiple donors, allelic dropout and stutters, 565 Forensic Sci Int Genet. 7 (2013) 555-563. DOI: 10.1016/j.fsigen.2013.05.009.

566 12. D. Taylor, J. Bright, J. Buckleton, The interpretation of single source and mixed DNA profiles,

567 Forensic Sci Int Genet. 7 (2013) 516-528. DOI: 10.1016/j.fsigen.2013.05.011.

568 13. P. Gill, J. Curran, C. Neumann, A. Kirkham, T. Clayton, J. Whitaker, J. Lambert, Interpretation 569 of complex DNA profiles using empirical models and a method to measure their robustness,

570 Forensic Sci Int Genet. 2 (2008) 91-103. DOI: 10.1016/j.fsigen.2007. 
571 14. G. Dørum, Ø . Bleka, P. Gill, H. Haned, L. Snipen, S. Sæbø, T. Egeland, Exact computation

572 of the distribution of likelihood ratios with forensic applications, Forensic Sci Int Genet. 9 (2014)

573 93-101. DOI: 10.1016/j.fsigen.2013.11.008.

574 15. M. Kruijver, Efficient computations with the likelihood ratio distribution, Forensic Sci Int Genet.

57514 (2015) 116-124. DOI: 10.1016/j.fsigen.2014.09.018.

576 16. D. Taylor, J. Buckleton, I. Evett, Testing likelihood ratios produced from complex DNA

577 profiles. Forensic Sci Int Genet. 16 (2015) 165-171. DOI: 10.1016/j.fsigen.2015.01.008.

578 17. M. Kruijver, R. Meester, K. Slooten, $p$-values should not be used for evaluating the strength of

579 DNA evidence. Forensic Sci Int Genet. 16 (2015) 226-231. DOI: 10.1016/j.fsigen.2015.01.005.

580 18. Ø. Bleka, G. Dørum, H. Haned, P. Gill, Database extraction strategies for low-template

581 evidence. Forensic Sci Int Genet. 9 (2014) 134-141. DOI: 10.1016/j.fsigen.2013.11.006.

582 19. J.M. Butler, Advanced Topics in Forensic DNA Typing: Interpretation, first ed., San Diego:

583 Elsevier Academic Press, 2014, pp. 159-177, 281-305, 349-387.

584 20. H. Swaminathan, C.M. Grgicak, M. Medard, D.S. Lun, NOClt: A computational method to infer 585 the number of contributors to DNA samples analyzed by STR genotyping, Forensic Sci Int Genet.

58616 (2015) 172-180. DOI: 10.1016/j.fsigen.2014.11.010.

587 21. C.M. Grgicak, Z.M. Urban, R.W. Cotton, Investigation of Reproducibility and Error Associated 588 with qPCR Methods using Quantifiler Duo DNA Quantitation Kit. J Forensic Sci. 55 (2010) 1331-

589 1339. DOI: $10.1111 / \mathrm{j} .1556-4029.2010 .01460 . x$.

590 22. M.C. Cicero, C.M. Grgicak, Examination into the Applicability and Stability of a Single External

591 Calibrator for Forensic DNA Quantification. NorthEastern Association of Forensic Scientists,

592 Cromwell, CT, 2013. Available at: http://www.bumc.bu.edu/gms/files/2013/03/NEAFS-Stability-of-

593 Validated-Curve-Abstract.pdf. More info: http://www.bumc.bu.edu/gms/biomedforensic/faculty-

594 and-staff/faculty/grgicak/tools.

595 23. Applied Biosystems, AmpFlstr ${ }^{\circledR}$ Identifiler ${ }^{\circledR}$ Plus PCR Amplification Kit Users' Manual, first ed., 5962006.

597 24. J.M. Butler, Fundamentals of forensic DNA typing, first ed., San Diego: Elsevier Academic

598 Press, 2009, pp. 36-37, 112.

599 25. D.J.C. Mackay, Information Theory, Inference and Learning Algorithms, Cambridge University 600 Press, 2003, p. 285-289. 
601 26. D.J.C. Mackay, Introduction to Monte Carlo Methods, Springer Netherlands, 1998, p. 361-

602364.

603 27. K. Slooten, R. Meester, Probabilistic strategies for familial DNA searching, J.R. Stat. Soc.:

604 Ser. C (Appl. Stat.) 63 (3) (2014) 361-384. DOI: 10.1111/rssc.12035.

605 28. D.R. Paoletti, T.E. Doom, C.M. Krane, M.L. Raymer, D.E. Krance, Empirical analysis of the

606 STR profiles resulting from conceptual mixtures, J Forensic Sci 50 (2005) 1361-1366.

607

608 FIGURES AND TABLES CAPTIONS

609 Table 1. The variables used in the study and the distribution used to model them as a function of

610 DNA mass. At each time of injection, different parameters were used for different loci.

611 Table 2. Genotypes of the contributors to the 3-person sample in Fig 2.

612 Table 3. Genotypes of the contributors to the 2-person sample in Fig 4.

613 Figure 1. LR from CEESIt for the contributors to the 1-, 2- and 3-person samples at the 10s

614 injection in the testing set. High LRs corresponded to high template DNA amounts. Contributors

615 with low template masses had high levels of dropout and stutter, resulting in small LRs. Similar

616 results were obtained at the other injection times.

617 Figure 2. Profile of a 3-person sample, amplified from 0.59ng of template DNA and a mixture ratio

618 of 1:9:9. Arrows represent alleles of minor contributor that had a height $<1$ RFU. The minor

619 contributor's alleles dropped out at 3 loci, leading to a low $\operatorname{LR}\left(10^{-7}\right)$ and a high $p$-value $\left(7^{*} 10^{-4}\right)$.

620 The major contributors had LRs of $10^{12}$ and $10^{11}$ and $p$-values of $10^{-9}$ and $10^{-9}$.

621 Figure 3. LR distribution from CEESIt for the sample in Fig 2. Most of the 1 billion samples had a

$622 \log (\mathrm{LR})$ less than 0 , with a peak at -30 .

623 Figure 4. Profile of a 2-person sample, amplified from $0.25 \mathrm{ng}$ of template DNA and a mixture ratio

624 of 1:2. The alleles of the minor contributor are well represented in the signal resulting in a LR of

$62510^{19}$ and a $p$-value of $10^{-9}$. The major contributor also had a LR of $10^{19}$ and a $p$-value of $10^{-9}$.

626 Figure 5. LR distribution from CEESIt for the sample in Fig 4. Many genotype samples had a

$627 \log (\mathrm{LR})$ less than 0. 481 million samples had a LR of 0 (and a Log(LR) of -Infinity). The plot

628 shows the distribution of the remaining 519 million samples..

629 Figure 6. The LR for a contributor with 0.0625ng template mass in a 1-, 2- and 3-person sample

630 at the 10 s injection. The contributor was the major in the 3 -person sample $(0.125 \mathrm{ng}$ total template 
631 mass, 1:2:1 mixture ratio), while the 2-person sample had a total template mass of $0.125 \mathrm{ng}$ and a $6321: 1$ mixture ratio. The LR decreased as the number of interference contributors increased.

633 Figure 7 (a-b). p-values from CEESIt as a function of LR for all the samples at the 10 s injection in

634 the testing set. All the 1-person samples had a $p$-value of $10^{-9}$. With regard to the 2-and 3-person

635 samples, the $p$-value was the lowest possible value of $10^{-9}$ when the LR was greater than $10^{8}$.

636 Similar results were obtained at the other injection times.

637 Figure 8. The results of 2 different runs on the 101, 1-, 2- and 3-person samples at the 10s

638 injection. There is very little variation from run to run; the points lie close to the $x=y$ diagonal line

639 with very few outliers (Pearson correlation coefficient $=0.9781$, slope $=1.01$, intercept $=0.03$ ).

640 Similar results were obtained at the other injection times.

641 Figure 9. LR for one 2-person sample (a) and two 3-person samples (b) using the true number of 642 contributors and an underestimate on the number of contributors.

643 Figure 10. LR for three 1-person samples (a) and two 2-person samples (b) using the true 644 number of contributors and an overestimate on the number of contributors.

645

646

647

648

649

650

651

652

653

654

655

656

657

658 
Figure 1

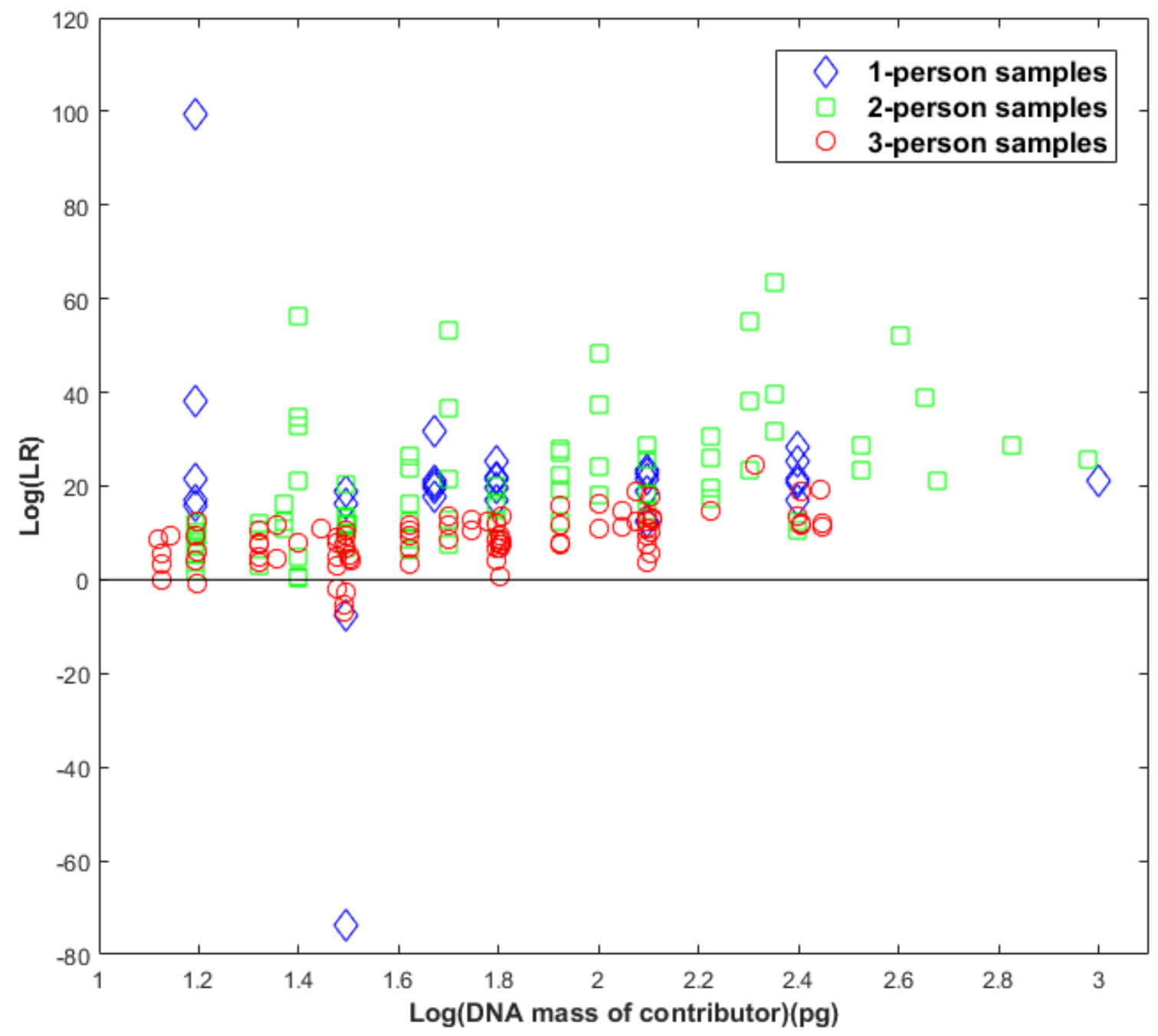


Figure 2

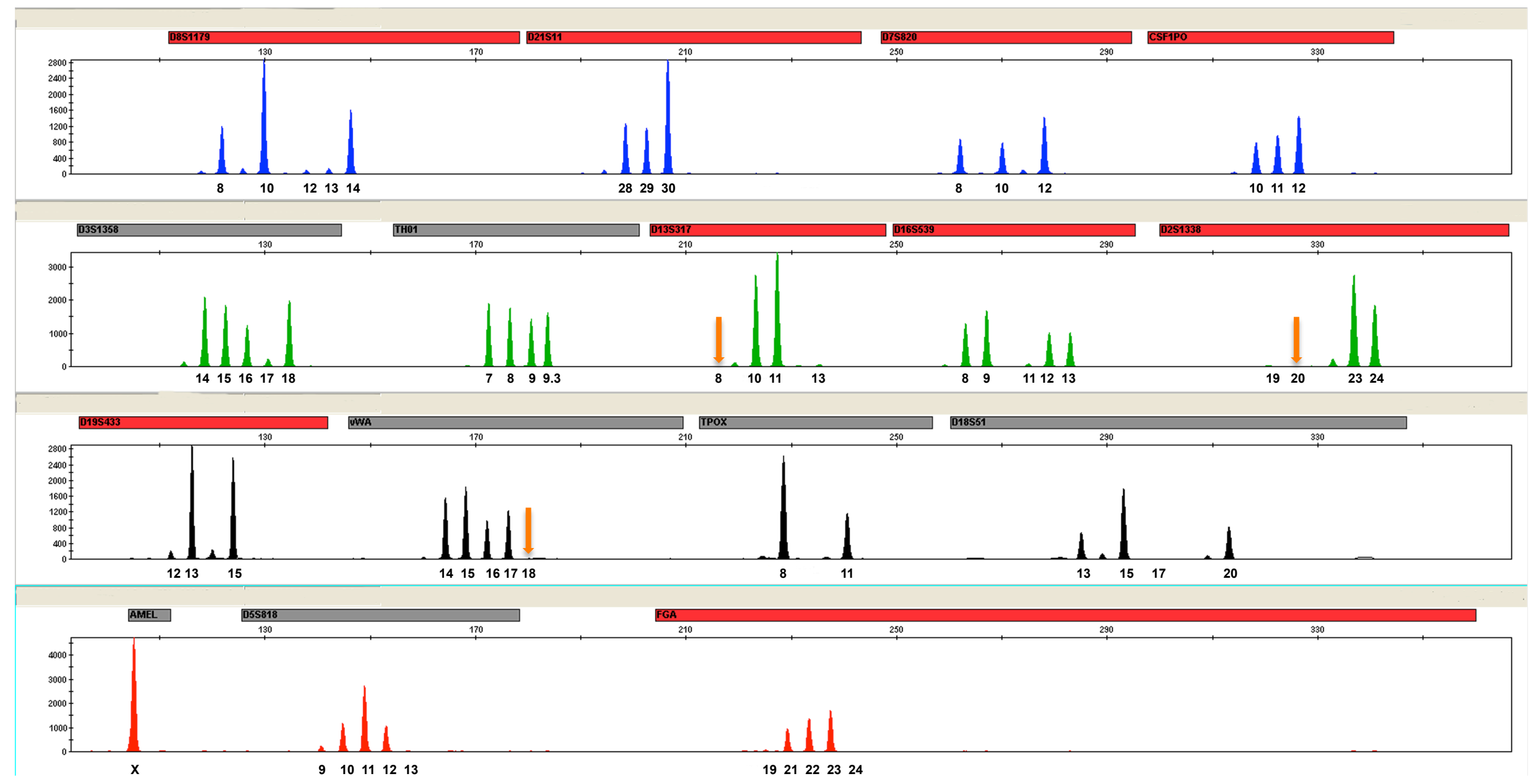

290 $\stackrel{330}{1}$ 


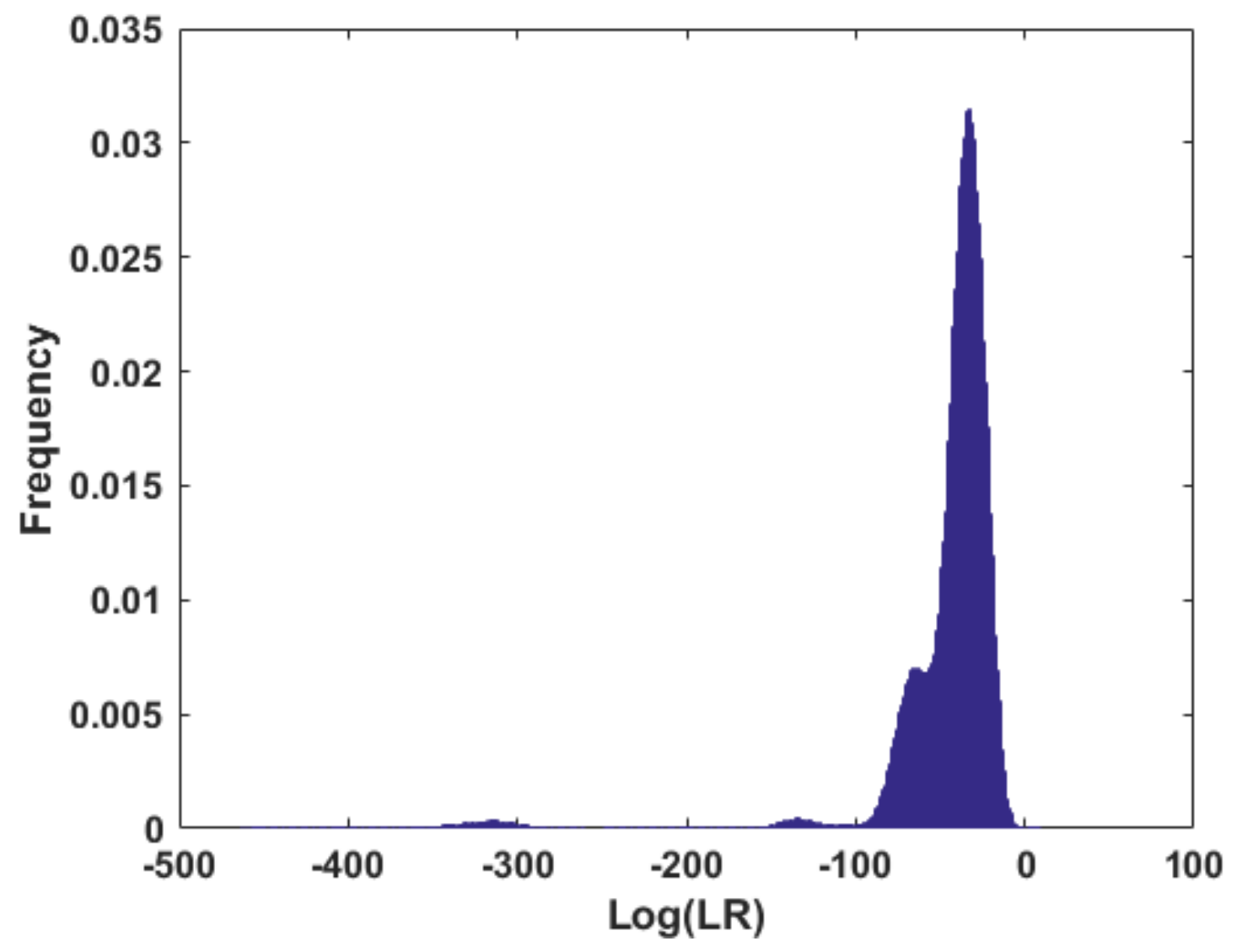


Figure 5

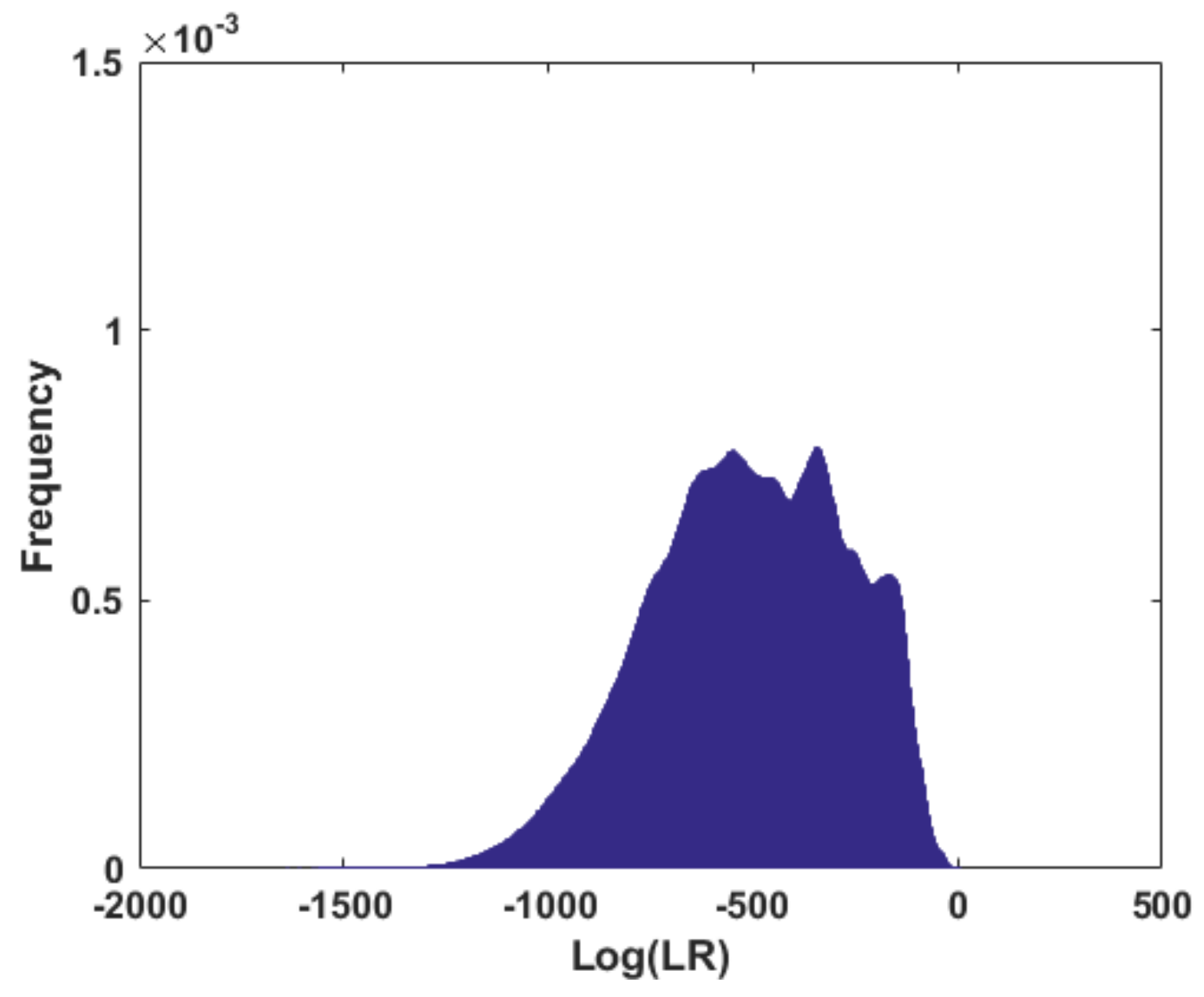


Figure 6

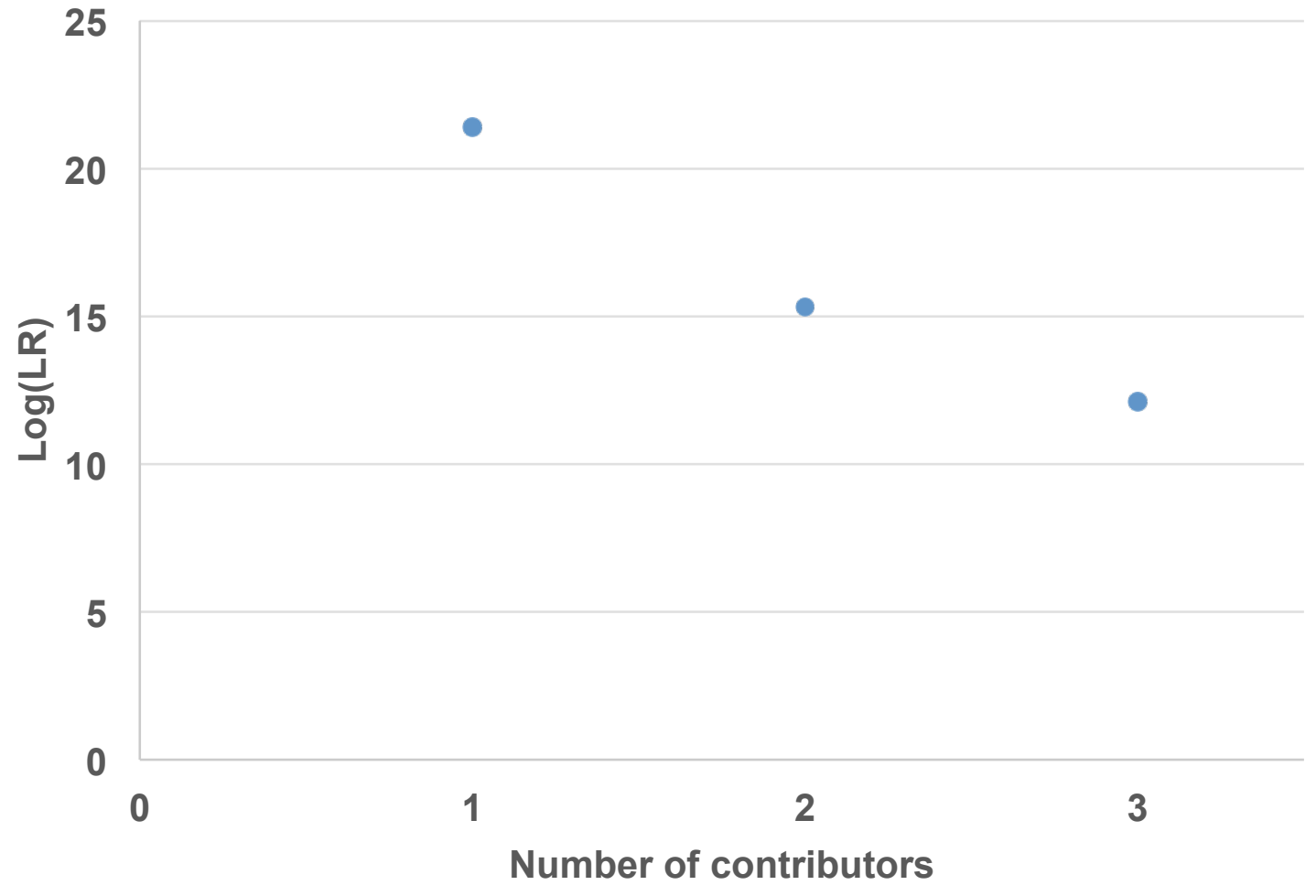




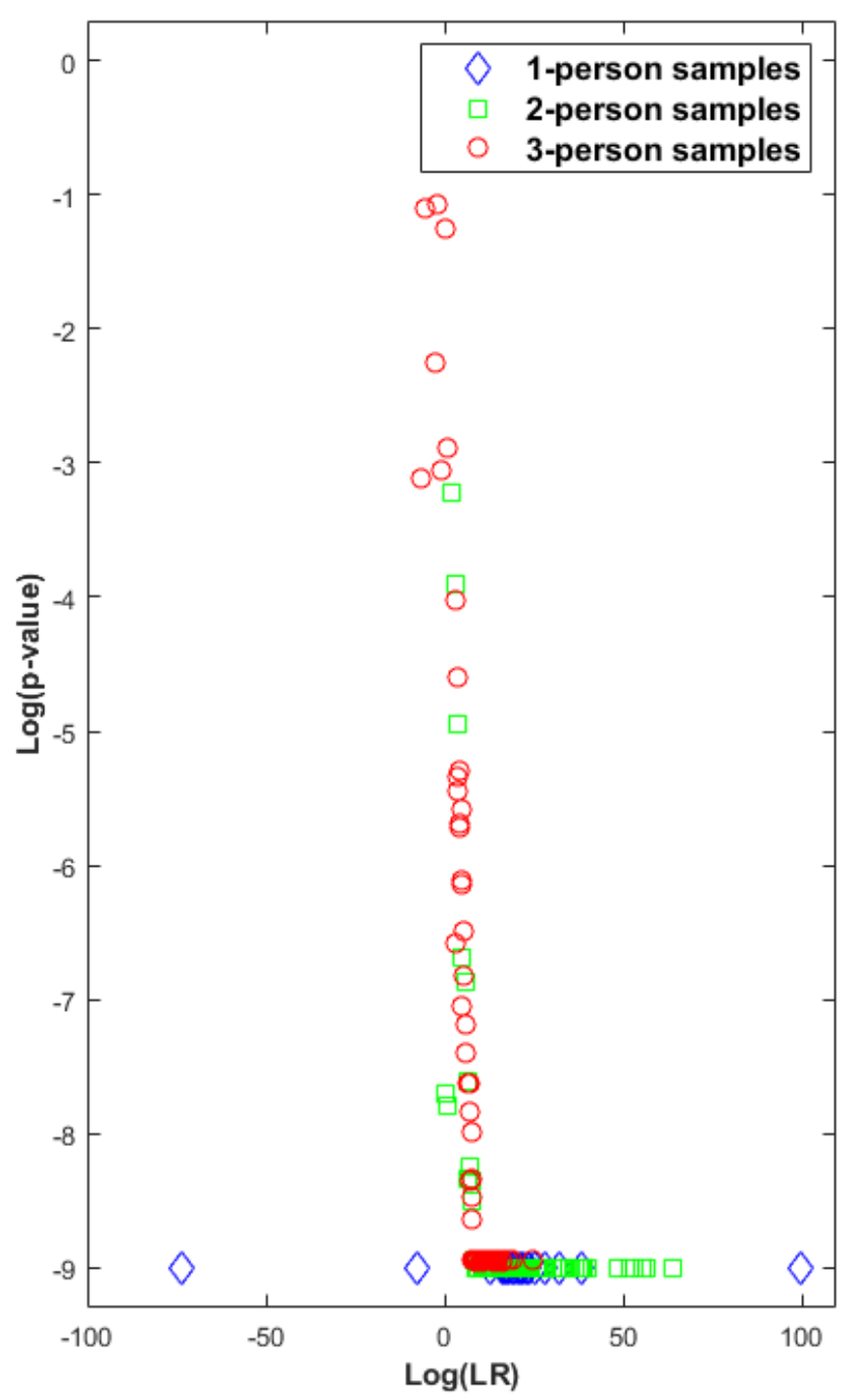

a

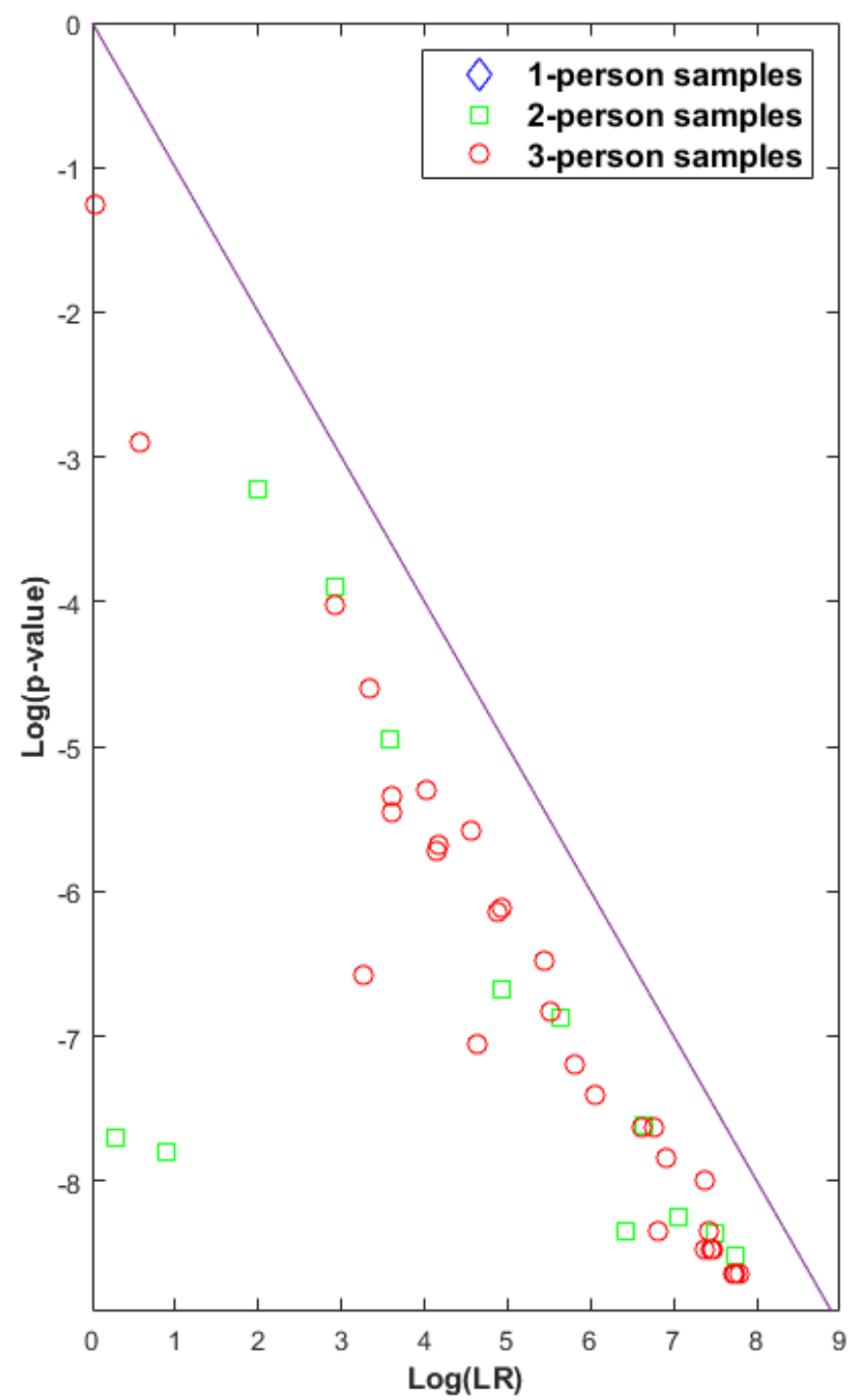

b 


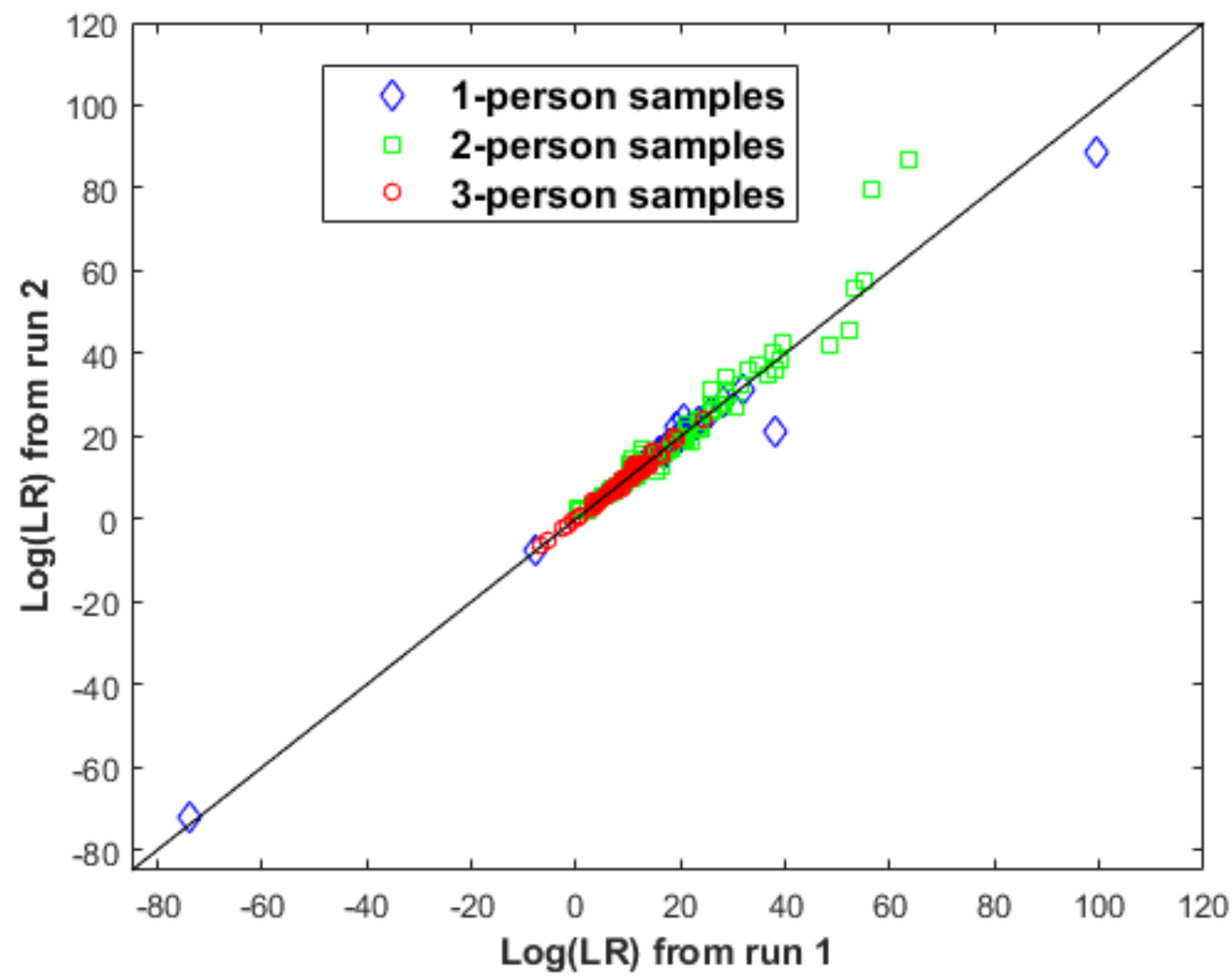




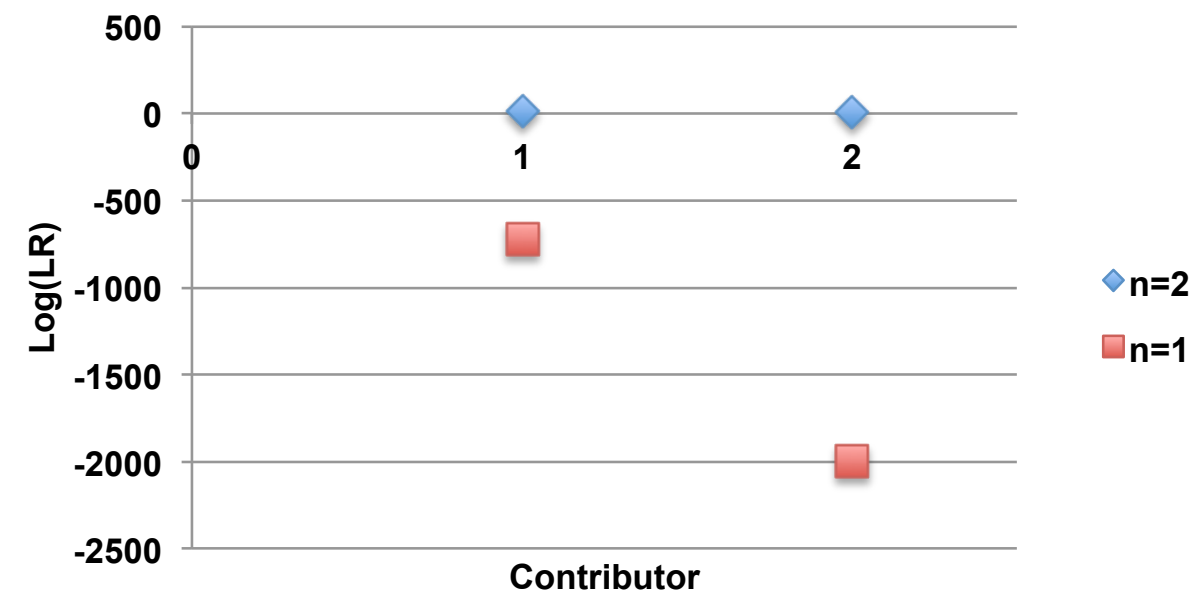

a
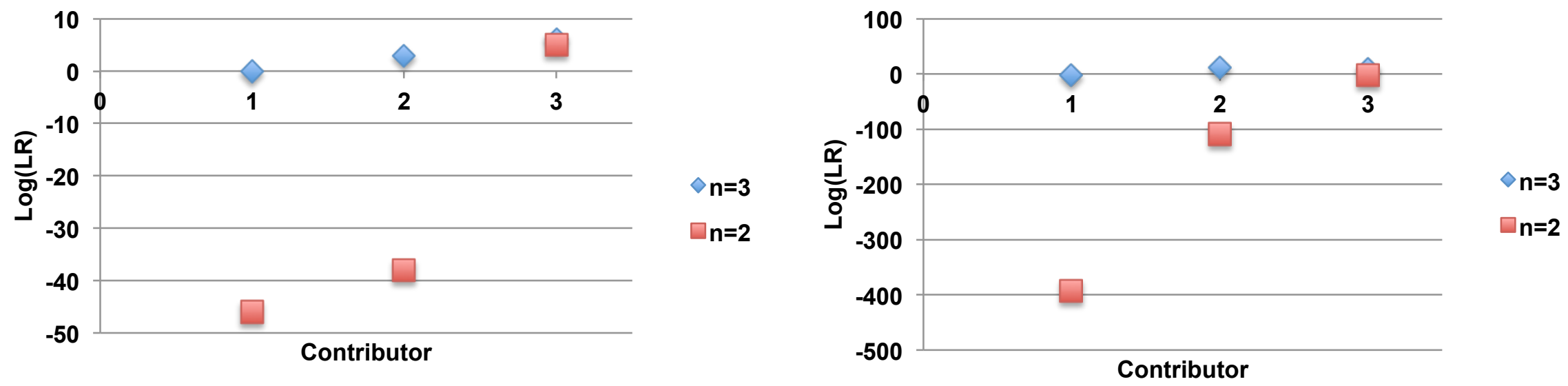

b 
Figure 10

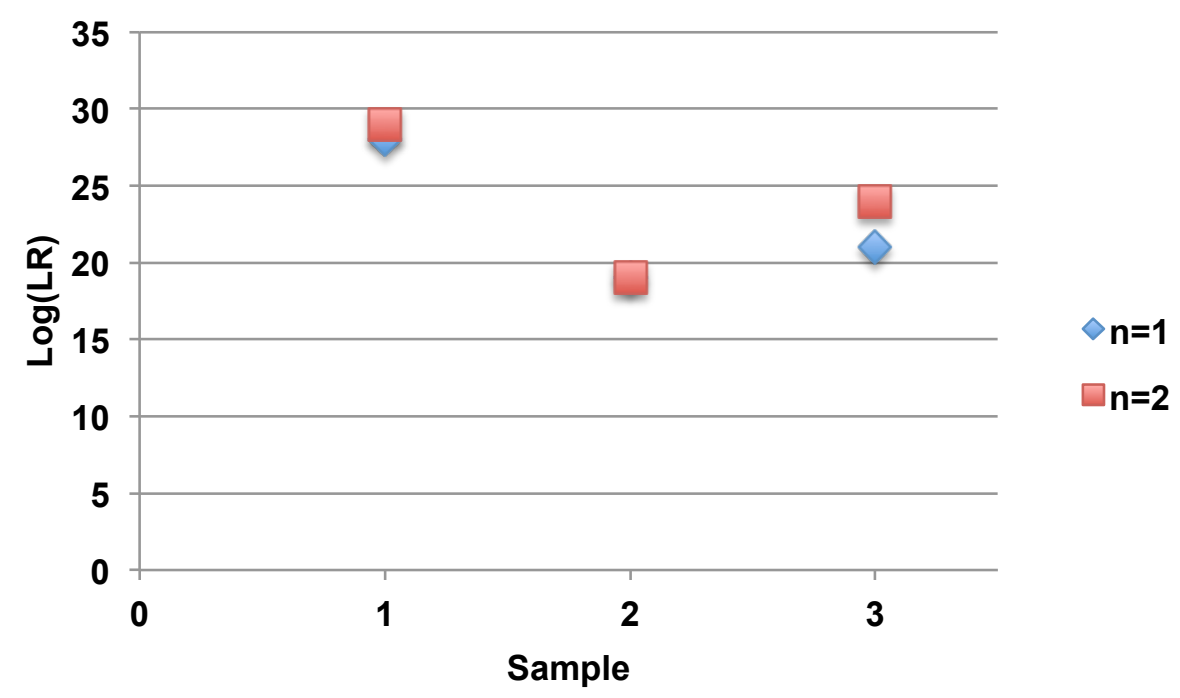

a
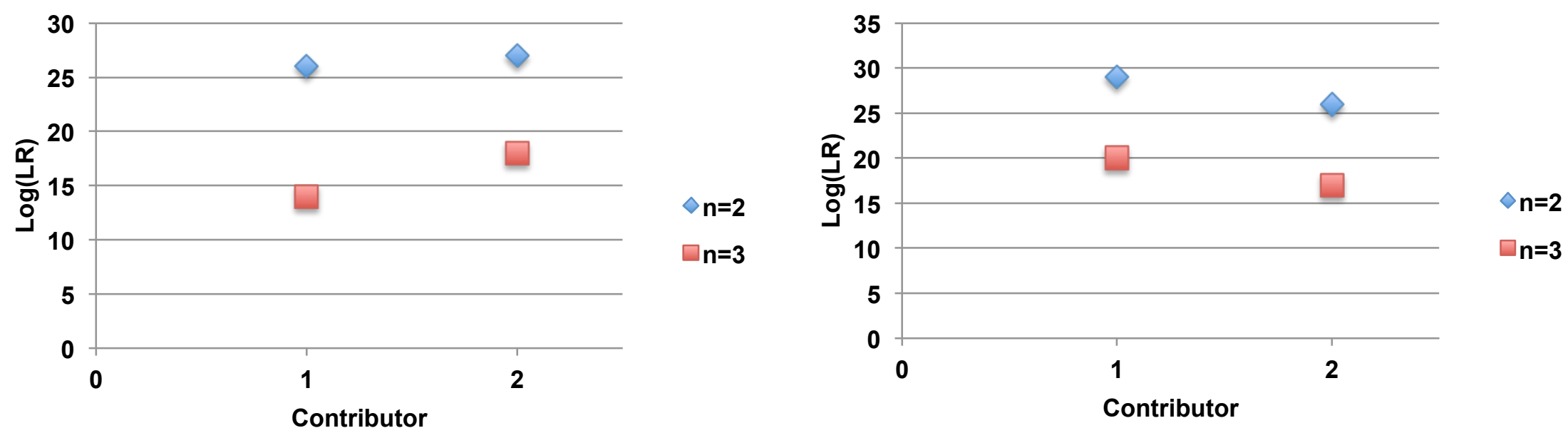

b 


\begin{tabular}{|c|c|c|}
\hline Variable & Model description & Model function \\
\hline Dropout rate of alleles $(\alpha)$ & $\begin{array}{l}\text { Exponentially decreasing } \\
\text { curve }\end{array}$ & $\begin{array}{l}\alpha=a e^{b x}, \text { where } x \text { is the DNA } \\
\text { mass from the contributor with } \\
\text { the allele }\end{array}$ \\
\hline $\begin{array}{l}\text { Rate of non-detection of } \\
\text { stutter }(\beta)\end{array}$ & $\begin{array}{l}\text { Exponentially decreasing } \\
\text { curve }\end{array}$ & $\begin{array}{c}\beta=a e^{b x}, \text { where } x \text { is the DNA } \\
\text { mass in the parent allele that } \\
\text { gives rise to stutter }\end{array}$ \\
\hline $\begin{array}{l}\text { Rate of non- detection of noise } \\
\qquad(\gamma)\end{array}$ & Constant & $\begin{array}{l}\text { Estimated from calibration } \\
\text { data } \\
\end{array}$ \\
\hline Mean of true peak heights $\left(\mu_{t}\right)$ & Line with a positive slope & $\begin{array}{l}\mu_{t}=a x+b, \text { where } x \text { is the } \\
\text { DNA mass in the true peak }\end{array}$ \\
\hline $\begin{array}{c}\text { Standard deviation of true } \\
\text { peak heights }\left(\sigma_{t}\right)\end{array}$ & Line with a positive slope & $\begin{array}{l}\sigma_{t}=a x+b, \text { where } x \text { is the } \\
\text { DNA mass in the true peak }\end{array}$ \\
\hline $\begin{array}{l}\text { Mean of noise peak heights } \\
\qquad\left(\mu_{n}\right)\end{array}$ & Line with a positive slope & $\begin{array}{c}\mu_{n}=a x+b, \text { where } x \text { is the } \\
\text { DNA mass that the sample } \\
\text { was amplified with }\end{array}$ \\
\hline $\begin{array}{l}\text { Standard deviation of noise } \\
\text { peak heights }\left(\sigma_{n}\right)\end{array}$ & Line with a positive slope & $\begin{array}{c}\sigma_{n}=a x+b, \text { where } x \text { is the } \\
\text { DNA mass that the sample } \\
\text { was amplified with }\end{array}$ \\
\hline Mean of stutter ratios $\left(\mu_{s}\right)$ & $\begin{array}{l}\text { Exponentially decreasing } \\
\text { curve }\end{array}$ & $\begin{array}{c}\mu_{s}=a e^{b x}+c, \text { where } x \text { is the } \\
\text { DNA mass in the parent allele } \\
\text { that gives rise to stutter }\end{array}$ \\
\hline $\begin{array}{l}\text { Standard deviation of stutter } \\
\text { ratios }\left(\sigma_{S}\right)\end{array}$ & $\begin{array}{l}\text { Exponentially decreasing } \\
\text { curve }\end{array}$ & $\begin{array}{c}\sigma_{s}=a e^{b x}+c, \text { where } x \text { is the } \\
\text { DNA mass in the parent allele } \\
\text { that gives rise to stutter }\end{array}$ \\
\hline
\end{tabular}




\begin{tabular}{|c|c|c|c|}
\hline Locus & Contributor 1 & Contributor 2 & Contributor 3 \\
\hline D8S1179 & 12,13 & 10,10 & 8,14 \\
\hline D21S11 & 28,30 & 30,30 & 28,29 \\
\hline D7S820 & 8,10 & 10,12 & 8,12 \\
\hline CSF1PO & 12,12 & 11,12 & 10,12 \\
\hline D3S1358 & 16,17 & 14,18 & 15,16 \\
\hline TH01 & 8,9 & $7,9.3$ & 8,9 \\
\hline D13S317 & 8,13 & 10,11 & 10,11 \\
\hline D16S539 & 11,12 & 9,12 & 8,13 \\
\hline D2S1338 & 19,20 & 23,24 & 23,24 \\
\hline D19S433 & 12,13 & 13,15 & 13,15 \\
\hline vWA & 15,18 & 15,17 & 14,16 \\
\hline TPOX & 8,11 & 8,8 & 8,11 \\
\hline D18S51 & 17,20 & 15,20 & 13,15 \\
\hline AMEL & $X, X$ & $X, X$ & $X, X$ \\
\hline D5S818 & 9,13 & 11,11 & 10,12 \\
\hline FGA & 19,24 & 22,23 & 21,23 \\
\hline
\end{tabular}




\begin{tabular}{|c|c|c|}
\hline Locus & Contributor 1 & Contributor 2 \\
\hline D8S1179 & 13,14 & 14,14 \\
\hline D21S11 & 27,29 & $30,30.2$ \\
\hline D7S820 & 8,12 & 8,9 \\
\hline CSF1PO & 10,12 & 12,12 \\
\hline D3S1358 & 16,18 & $15,18.2$ \\
\hline TH01 & 7,8 & $7,9.3$ \\
\hline D13S317 & 12,14 & 11,13 \\
\hline D16S539 & 8,9 & 9,11 \\
\hline D2S1338 & 17,17 & 22,24 \\
\hline D19S433 & 13,13 & 11,13 \\
\hline vWA & 17,19 & 15,17 \\
\hline TPOX & 9,10 & 8,8 \\
\hline D18S51 & 15,16 & 14,17 \\
\hline AM & $X, Y$ & $\times, Y$ \\
\hline D5S818 & 12,12 & 11,11 \\
\hline FGA & 22,24 & 22,23 \\
\hline
\end{tabular}

